\title{
HORIZONTAL MARTINGALES IN VECTOR BUNDLES
}

\author{
MARC ARNAUDON AND ANTON THALMAIER*
}

\begin{abstract}
Canonical prolongations of manifold-valued martingales to vector bundles over a manifold are considered. Such prolongations require a lift of the connection from the manifold to the corresponding bundle. Given a continuous semimartingale $X$ in $M$, if $\nabla$ is a connection on $M$ (i.e. a covariant derivative on $T M$ ) and $\nabla^{\prime}$ the lifted connection on $E$ (i.e. a covariant derivative on $T E$ ), we consider semimartingales $J$ in $E$, living above $X$ and linked to $X$ via $d^{\nabla^{\prime}} J=h_{J}\left(d^{\nabla} X\right)$ where $h_{J}: T_{X} M \rightarrow T_{J} E$ is the horizontal lift; $d^{\nabla} X$ and $d^{\nabla^{\prime}} J$ denote the Itô differentials with respect to the given connection. Such semimartingales $J$ in $E$ will be called horizontal semimartingales, resp. horizontal martingales in case when $X$ is a $\nabla$-martingale. There are numerous ways of lifting $\nabla$ to $\nabla^{\prime}$. We mainly deal with horizontal and complete lifts. Horizontal lifts give rise to the notion of covariant Itô differentials. For covariant Itô differentials a commutation formula with ordinary covariant differentials is established. As an application, covariant variations of stochastic parallel transport and their relation to Yang-Mills connections are investigated. On the other side, within the framework of complete lifts of connections, the martingale property is preserved under taking derivatives (or exterior derivatives) of families of martingales and is inherited to diffusions on the exterior cotangent bundle with the de Rham-Hodge Laplacian as generator. Moreover, in a natural way, derivatives of harmonic maps are harmonic as well.
\end{abstract}

\section{CONTENTS}

1. Introduction and Notations

2. Complete Lifts to Tangent Bundles 5

3. Deformed Antidevelopment 7

4. Horizontal Lifts to Vector Bundles 9

5. A General Class of Lifts to Vector Bundles 20

6. Complete Lifts to Cotangent Bundles 26

7. Complete Lifts to Exterior Bundles 27

8. Complete Lifts to Dirac Bundles 31

9. Martingales in the Tangent Space Related to Harmonic Maps 33

10. Martingales in the Exterior Tangent Bundle Related to Harmonic Forms 36

$\begin{array}{ll}\text { References } & 38\end{array}$

* Research supported by Deutsche Forschungsgemeinschaft and SFB 256 (University of Bonn). 


\section{InTRODUCTION AND NOTATIONS}

Let $M$ be a differentiable manifold of dimension $m$, endowed with a linear connection

$$
\nabla: \Gamma(T M) \rightarrow \Gamma\left(T^{*} M \otimes T M\right), \quad \nabla_{V} W \equiv(\nabla W) V,
$$

and let $\pi: E \rightarrow M$ be a vector bundle over $M$. Typically, $E$ will be the tangent bundle $T M$, the cotangent bundle $T^{*} M$, the exterior tangent bundle $\wedge T M$, resp., cotangent bundle $\wedge T^{*} M$, or more generally, some Dirac bundle over $M$. Let $F_{0}$ be a vector space isomorphic to the typical fiber of $E$. We denote by $\pi: L\left(F_{0}, E\right) \rightarrow M$ the vector bundle of linear maps $F_{0} \rightarrow E_{x}, x \in M$. In other words, $L\left(F_{0}, E\right)=F_{0}^{*} \otimes E$ when $F_{0}$ is considered as the trivial bundle over $M$ with fiber $F_{0}$. Let $L(M)=L\left(\mathbb{R}^{m}, T M\right)$.

In this article we shall deal with the following problem: Given a $\nabla$-martingale $X$ in $M$, we are interested in lifting $X$ from $M$ to $L\left(F_{0}, E\right)$ in such a way that the lifted process $W$ above $X$ (i.e., $\pi \circ W=X$ ) yields a martingale taking values in the vector bundle $L\left(F_{0}, E\right)$, and such that there is a one to one correspondence $Z \mapsto W Z$ between the local martingales in $F_{0}$ and the martingales in $E$ which project onto $X$. This clearly requires a connection on $E$ as an additional structure, i.e. a covariant derivative on $T E$,

$$
\nabla: \Gamma(T E) \rightarrow \Gamma\left(T^{*} E \otimes T E\right) .
$$

The problem of lifting martingales and connections to vector bundles over a manifold has already been studied in [16] where horizontal and complete lifts to vector bundles were investigated. Extending our work in the case $E=T M$ [2], we focus here on various properties of horizontal and complete lifts, in particular in relation to the variation of families of semimartingales. We differentiate martingales with respect to a parameter, take exterior products, to obtain martingales for complete lifts of connections. Finally we relate our results to the theory of diffusion operators and stochastic flows, as developed in [10].

Our original motivation comes from the following problem: For functions $f \in$ $C^{\infty}(M)$ on a Riemannian manifold $M$ it is well-known that $f$ is harmonic (i.e., $\Delta f=0$ where $\Delta$ is the Laplace-Beltrami operator on $M$ ) if and only if the composition $f \circ X$ with Brownian motions $X$ on $M$ provides real-valued local martingales. Instead of functions $f$, one may consider differential forms $\alpha \in \Gamma\left(\wedge T^{*} M\right)$. We like to find a connection $\nabla$ on $E=\wedge T^{*} M$ in the sense of (1.2), such that a similar characterization holds true: $\alpha$ is harmonic (i.e., $\Delta \alpha=0$ where $\Delta$ is the Hodge-de Rham operator on forms) if and only if $\alpha \circ X$ is an $E$-valued $\nabla$-martingale for any Brownian motion $X$ on $M$. More generally, if $E$ is a Dirac bundle and $D$ its Dirac operator, we investigate connections $\nabla$ on $E$ such that $a \in \Gamma(E)$ is harmonic (i.e., $\Delta a:=-D^{2} a=0$ ) if and only if all compositions $a \circ X$ with Brownian motions $X$ give $\nabla$-martingales in $E$.

These characterizations do not determine the connection in a unique way, as long as we consider only martingales which project to Brownian motions on $M$. As well-known, the full class of $\nabla$-martingales determines the connection up to torsion.

The paper is organized as follows. In Section 2 we recall some results concerning complete lifts of connections to tangent and frame bundles. Theorem 2.1 ([16] Corollaire 8) gives a one to one correspondence $Z \mapsto \Theta Z$ between the set of local martingales in a tangent space $T_{X_{0}} M$ and the set of martingales in the tangent bundle $T M$ which project onto a given martingale $X$ in the manifold; here $\Theta$ is not the usual parallel transport but the geodesic transport along $X$, see Eq. (2.1) below. Theorem 2.2 ([3] 
Corollary 3.14) guarantees that Itô stochastic differential equations between manifolds differentiate like ordinary equations, if the tangent bundles are equipped with the complete lifts of the connections in the manifolds. Corollary 2.3 ([3] Theorem 4.1 and [2] Theorem 3.1) gives a commutation formula between antidevelopment and differentiation with respect to a parameter, and an interpretation of martingales in the tangent bundle as stochastic Jacobi fields, i.e. as derivatives of families of martingales living in the manifold.

In Section 3 we continue to work on the tangent bundle of $M$. The main result is Theorem 3.1 which says that the commutation formula for antidevelopment and derivation (Corollary 2.3) still holds true if parallel transport is replaced by deformed parallel transport (i.e. geodesic transport). Again the key step in the proof is Theorem 2.2.

In Section 4 we consider the situation where a connection $\nabla$ on $M$ and a covariant derivative $\nabla^{E}$ on the vector bundle $E$ is given. It is well known that there is a canonical induced connection $\nabla^{h}$ on $E$, called the horizontal lift of $\nabla$ and $\nabla^{E}$. Here we give characterizations of martingales, parallel transport and antidevelopment with respect to $\nabla^{h}$. In particular, a covariant Itô differential of an $E$-valued semimartingale is defined. The main result is a commutation formula for the covariant Itô differential and the covariant derivative with respect to a parameter (Theorem 4.5). Various applications related to Yang-Mills connections, and in relation to parallel transport along rescaled Brownian bridge are given. In particular, the covariant derivative of parallel transport along a Brownian motion is shown to play the same role in the theory of Yang-Mills connections, as the image of a Brownian motion under a harmonic map in the theory of harmonic maps. Also we write a Taylor expansion up to order 3 of the parallel transport along a rescaled Brownian bridge, and prove that for Yang-Mills connections all the drifts of the terms of the expansion vanish (Theorem 4.13).

Section 5 is devoted to the study of a class of connections on $E$ which differ from a horizontal lift by a bilinear map similar to the one we obtain when considering complete lifts on $T M$. We describe again martingales, parallel transport, antidevelopment. This extends Meyer's results in [16] to connections with torsion on general vector bundles. Note that for Le Jan-Watanabe connections, which are connections considered in most of the examples given here, the torsion term cannot be suppressed. We discuss the construction of dual connections in dual bundles and tensor products. When $M$ is a Riemannian manifold, a Laplacian on $E$ associated with the considered connection is defined, and a stochastic interpretation to the heat equation on $E$ (Theorem 5.8 and Corollary 5.9) is derived.

In Sections 6, 7 and 8 examples of connections constructed in Section 5 are given. In Section 6 we consider the Levi-Civita connection $\nabla^{b}$ to the natural pseudo-metric $2 d x^{i} \nabla p_{i}$ in the cotangent bundle $T^{*} M$. It is not the dual connection to the complete lift in $T M$, as discussed in Section 5. However we remark that when $M$ is a Riemannian manifold equipped with the Levi-Civita connection, then the natural map $\left(T M, \nabla^{c}\right) \rightarrow$ $\left(T^{*} M, \nabla^{b}\right)$ is affine.

In Section 7 we define connections $\nabla^{c}$ on the exterior bundles $\wedge^{p} T M$ and by duality on $\wedge^{p} T^{*} M$, which we call complete lifts of $\nabla$. They naturally extend the definitions in tangent and cotangent bundles. When $M$ is a Riemannian manifold, the trace of the bilinear map added to the horizontal lift is the term arising in Weitzenböck's formula. 
However we are interested in the more general context of Le Jan-Watanabe connections $\nabla$ (Definition 7.5) associated with diffusion processes $X$ on $M$. The main result of this section (Proposition 7.6) says that if $X$ is a $\nabla$-martingale then $T X^{\wedge p}$ is a $\nabla^{c}$ martingale. This is in fact a consequence of a formula established in the proof of Theorem 3.3.8 in [10], and can be seen as some kind of extension of the stochastic Jacobi field representation of martingales in the tangent bundle. The principal argument of our proof is Corollary C.5 in [10]. Besides the stochastic Jacobi field representation, the aim is to translate the geometric theory of diffusions on manifolds as developed in Elworthy-Le Jan-Li in [10] into the language of martingales in manifolds.

In Section 8 we deal with complete lifts to Dirac bundles. Again the problem is to find a bilinear map which gives the complete lift when added to the horizontal lift, and such that its trace is the Weitzenböck term. There is a natural candidate built from the Clifford action, which in the particular case of the exterior bundle however does not respect the graduation, so it does not generalize the complete lift of Section 7. If the Clifford action is the difference of a creation and an annihilation operator, another natural candidate differs from the complete lift in Section 7 by an antisymmetric term, so both connections yield the same class of martingales.

In Section 9 we prove that the derivative of an $L$-harmonic map $u$ between manifolds $M$ and $\left(N, \nabla^{N}\right)$ is a harmonic map $M \rightarrow T^{*} M \otimes T N$ if $T^{*} M \otimes T N$ is endowed with a complete lift $\nabla^{c}$ constructed along the lines of Section 5, under the condition that there exists a Le Jan-Watanabe connection $\nabla^{M}$ on $M$ induced from the second order generator $L$ (Corollary 9.2). The condition is satisfied when $M$ is a Riemannian manifold and $\nabla^{M}$ its Levi-Civita connection. Under a slightly more restrictive assumption, Corollary 9.2 could also be proved by means of the method of filtering out redundant noise as in Elworthy-Le Jan-Li ([10]). Here we give a proof based on a geometric Itô formula involving complete lifts of connections. Corollary 9.2 can be seen as a generalization of the derivation property for geodesics; it illustrates in particular the interplay between complete lifts and derivations.

In Section 10 we consider some kind of inverse problem to the one of Section 9. Here we are given an $L$-harmonic $p$-form $\alpha$ where $L$ is a second order generator, we know that $\langle\alpha, \Theta\rangle$ is a local martingale where $\Theta$ is a horizontal martingale as defined in Section 5, and we want to prove that $\left\langle\alpha, T X^{\wedge p}\right\rangle$ is a local martingale if $X$ is a diffusion with generator $L$. Proposition 10.1 says that this is true under some Le Jan-Watanabe condition. Once more this is an illustration of the interaction between complete lifts and derivations. Proposition 10.1 is due to Elworthy-Li-Le Jan ([10] Theorem 2.4.2). We give an alternative proof relying on Itô's formula.

Throughout the paper we adopt the following conventions. By a connection on a manifold $M$ we mean a covariant derivative on $T M$ as in Eq. (1.1). The manifold $M$ may be a vector bundle $E$ as well, then a connection on $E$ will be a covariant derivative on $T E$ in the sense of Eq. (1.2). In many cases $E$, considered as vector bundle over $M$, may carry a connection

$$
\nabla^{E}: \Gamma(E) \rightarrow \Gamma\left(T^{*} M \otimes E\right)
$$

We refer to this as a covariant derivative on $E$.

A covariant derivative on $E$ gives rise to the splitting of the bundle $T E$ into the sum of a horizontal bundle and the vertical bundle. The covariant derivative of a smooth path 
$t \mapsto e(t) \in E$, denoted by $\nabla_{D} e$, will be $v_{e}^{-1}\left(\dot{e}^{\text {vert }}\right)$ where $\dot{e}^{\text {vert }}$ is the vertical part of $\dot{e}$ in this splitting, and $v_{e_{0}}: E_{\pi\left(e_{0}\right)} \rightarrow T_{e_{0}} E$ is the vertical lift to $e_{0} \in E$. Let $F_{0}$ be a vector space isomorphic to the typical fiber of $E$. Then a covariant derivative $\nabla^{E}$ on $E$ naturally induces a covariant derivative on $L\left(F_{0}, E\right)$, again denoted by $\nabla^{E}$, and defined by $\left(\nabla^{E} W\right)(w)=\nabla^{E}(W(w))$ where $w \in F_{0}$ and $W$ is a section of $L\left(F_{0}, E\right)$.

If $X$ is an $M$-valued continuous semimartingale and $\alpha$ is a section of $T^{*} M$, we denote by $\int_{0}\langle\alpha, \delta X\rangle$ the Stratonovich integral of $\alpha$ along $X$, and by $\int_{0}\left\langle\alpha, d^{\nabla} X\right\rangle$ the Itô integral. Recall that $X$ is a $\nabla$-martingale if and only if, for every $\alpha$ as above, $\int_{0}\left\langle\alpha, d^{\nabla} X\right\rangle$ is a local martingale. In local coordinates, we have

$$
\langle\alpha, \delta X\rangle=\alpha_{i}(X) d X^{i}+\frac{1}{2} \frac{\partial \alpha_{i}}{\partial x^{j}}(X) d<X^{i}, X^{j}>
$$

and

$$
\left\langle\alpha, d^{\nabla} X\right\rangle=\alpha_{i}(X)\left(d X^{i}+\frac{1}{2} \Gamma_{j k}^{i}(X) d<X^{j}, X^{k}>\right)
$$

where $\Gamma_{j k}^{i}$ are the Christoffel symbols of $\nabla$. Given a covariant derivative $\nabla^{E}$ on $E$, the parallel transport in $E$ along $X$ is the $L\left(E_{X_{0}}, E\right)$-valued semimartingale $/ /_{0, .}^{E}$ satisfying

$$
/ /_{0,0}^{E}=\operatorname{id}_{E_{X_{0}}} \quad \text { and } \quad \delta / /_{0, t}^{E}=h_{/ /_{0, t}^{E}}\left(\delta X_{t}\right)
$$

where $h_{e}: T_{\pi(e)} M \rightarrow T_{e} L\left(E_{X_{0}}, E\right), e \in L\left(E_{X_{0}}, E\right)$ denotes the horizontal lift associated to $\nabla^{E}$. Note that $/ /_{0, t}^{E} \in L\left(E_{X_{0}}, E_{X_{t}}\right)$.

Let $L$ be a second order generator on $M, N$ be another manifold endowed with a connection $\nabla^{N}$. We say that a smooth map $u:(M, L) \rightarrow\left(N, \nabla^{N}\right)$ is harmonic if $u(X)$ is a $\nabla^{N}$-martingale for every diffusion $X$ in $M$ with generator $L$.

\section{Complete Lifts to Tangent Bundles}

Let $M$ be a manifold equipped with a connection $\nabla$. In this section we recall some facts for the complete lift of connections to the tangent bundle of $M$, the local characteristics of $T M$-valued semimartingales and the horizontal $T M$-valued martingales. In particular we describe the links between complete lift and derivation. We refer to [19] for the geometric objects.

For a smooth function $f$ on $M$, the one-form $d f$ can be considered as a function on $T M$. This function is called the complete lift of $f$ and denoted by $f^{c}$. Every vector field $A \in \Gamma(T M)$ on $M$ induces a vector field $A^{c} \in \Gamma(T T M)$ on $T M$ which is characterized by the property $A^{c}\left(f^{c}\right)=(A(f))^{c}$ and called the complete lift of $A$. It is shown in [19] that the formula $\nabla_{A^{c}}^{c} B^{c}=\left(\nabla_{A} B\right)^{c}$, where $A, B \in \Gamma(T M)$, defines a connection $\nabla^{c}$ on $T M$, called the complete lift of $\nabla$.

Let $w \in T T M$. There exists a smooth path $t \mapsto u(t) \in T_{x(t)} M$, where $x(t)=$ $\pi\left(u(t)\right.$ ), such that $w=\dot{u}(0)$. We say that $w$ is horizontal if $\nabla_{\dot{x}(0)} u=0$ (note that this definition differs from the definition in [19] when the connection has torsion). Every vector splits into a horizontal and a vertical part, and for $u \in T_{x} M$ we denote by $h_{u}$ (resp. $v_{u}$ ) the horizontal (resp. vertical) lift $T_{x} M \rightarrow T_{u} T M$.

If $X$ is an $M$-valued semimartingale, the deformed parallel translation along $X$ (also called Dohrn-Guerra transport or geodesic transport in [16]), denoted by $\Theta_{0, t}$, is a linear 
map $T_{X_{0}} M \rightarrow T_{X_{t}} M$ such that $t \mapsto \Theta_{0, t}$ is a semimartingale and solution to the Itô equation

$$
\Theta_{0,0}=\operatorname{id}_{T_{X_{0}} M}, \quad d^{\nabla^{c}} \Theta_{0, t}=h_{\Theta_{0, t}}\left(d^{\nabla} X_{t}\right)
$$

(here $\nabla^{c}$ is considered as a connection on $L\left(T_{X_{0}} M, T M\right)$ ).

The $T M$-valued continuous $\nabla^{c}$-martingales were first considered by Meyer in [16], in the case where $\nabla$ is torsion-free. He obtained the following characterization:

Theorem 2.1. Assume that $\nabla$ is torsion-free. Let $J$ be a $T M$-valued semimartingale with projection $X$. Then $J$ is a $\nabla^{c}$-martingale if and only if

(i) $X$ is a $\nabla$-martingale, and

(ii) $\Theta_{0, .}^{-1} J$ is a $T_{X_{0}} M$-valued local martingale.

Now we investigate the relations between complete lift and derivation. Let $N$ be another manifold equipped with a connection, also denoted $\nabla$, and let $e$ be a smooth section of $T^{*} M \otimes T N$ over $M \times N$ (i.e., $e(x, y)$ is a linear map $T_{x} M \rightarrow T_{y} N$ for $(x, y) \in M \times N)$. The following result has been proved in [3]:

Theorem 2.2. Let $X(a)$ be a family of $M$-valued semimartingales which is $C^{1}$ in $a \in I$ with respect to the topology of semimartingales, where $I$ is some open interval in $\mathbb{R}$, and let $Y_{0}(a)$ be a family of $N$-valued random variables, $C^{1}$ in $a \in I$ with respect to the topology of convergence in probability.

Then the solution $Y(a)$, starting at $Y_{0}(a)$, to

$$
d^{\nabla} Y(a)=e(X(a), Y(a)) d^{\nabla} X(a)
$$

is $C^{1}$ in a with respect to the topology of semimartingales, its derivative $\partial Y(a)$ starts from $\partial Y_{0}(a)$ and is solution to

$$
d^{\nabla^{c}} \partial Y(a)=e^{\prime}(\partial X(a), \partial Y(a)) d^{\nabla^{c}} \partial X(a)
$$

where $e^{\prime}$ is determined by taking for $X$ a family of smooth deterministic paths.

Note that in [3] the topology of semimartingales is defined on processes with random lifetime.

Taking as a special case the antidevelopment $Z(a)=\mathscr{A}(X(a))$ of $X(a)$,

$$
Z(a)=\int_{0} / /_{0, s}^{-1}(a) d^{\nabla} X_{s}(a)
$$

where $/ /_{0, t}(a)$ is the parallel transport along $X(a)$, the following corollary has been obtained:

Corollary 2.3. (i) If $a \mapsto X(a)$ is $C^{1}$ in the topology of semimartingales, then

$$
\partial \mathscr{A}(X(a))=s_{M} \mathscr{A}^{c}(\partial X(a))
$$

where $\mathscr{A}^{c}$ is the antidevelopment with respect to $\nabla^{c}$ and $s_{M}: T T M \rightarrow T T M$ denotes the canonical involution (see below before Theorem 3.1 for the precise definition).

(ii) A semimartingale $J$ with values in $T M$ is $a \nabla^{c}$-martingale if and only if it is of the form $\partial X(0)$ where $a \mapsto X(a)$ is a family of $\nabla$-martingales, $C^{1}$ in the topology of semimartingales.

Corollary 2.3 (ii) gives an interpretation of $\nabla^{c}$-martingales as stochastic Jacobi fields. 


\section{Deformed Antidevelopment}

In this section the manifold $M$ is assumed to be equipped with a torsion-free connection $\nabla$. Let $X$ be an $M$-valued semimartingale. Define the deformed antidevelopment of $X$ as the $T_{X_{0}} M$-valued semimartingale

$$
\mathscr{A}_{\mathrm{def}}(X):=\int_{0} \Theta_{0, s}^{-1} d^{\nabla} X_{s}
$$

where $\Theta_{0, .}$ is the deformed parallel translation along $X$ as defined in Section 2. Note that by [12] Lemma (8.24), the Itô integral can be replaced by a Stratonovich integral. In this section we show that Eq. (2.2) remains true when antidevelopments are replaced by deformed antidevelopments.

We first collect some notations. Let $m$ be the dimension of $M$.

As in [2], for a manifold $F$, let $s_{F}: T T F \rightarrow T T F$ be the canonical isomorphism described as follows: if $v=\partial_{a} \partial_{t} x(t, a)$ for some smooth map $(t, a) \mapsto x(t, a) \in F$, then $s_{F}(v)=\partial_{t} \partial_{a} x(t, a)$. Denote by $L(F)$ the frame bundle over $F$.

A map $\imath_{M}: T L(M) \rightarrow L(T M)$ is given as follows: let $W=\dot{u}(0) \in T L(M)$ for some smooth curve $a \mapsto u(a) \in L(M)$ and $v=\dot{b}(0) \in T \mathbb{R}^{m}=\mathbb{R}^{2 m}$ for some smooth curve $a \mapsto b(a) \in \mathbb{R}^{m}$, then

$$
\imath_{M}(W) v=s_{M}\left((u b)^{\cdot}(0)\right) .
$$

The map $\imath_{\mathrm{GL}(m, \mathbb{R})}$ will be denoted by $\imath_{m}$. Further let

- $\tilde{\pi}$ be the canonical projection $L(T M) \rightarrow T M$,

- $\omega\left(\right.$ resp. $\left.\omega^{c}\right)$ the connection form associated with $\nabla$ (resp. $\left.\nabla^{c}\right)$,

- $\vartheta \in \Gamma\left(T^{*} L(M) \otimes \mathbb{R}^{m}\right)\left(\right.$ resp. $\vartheta^{c} \in \Gamma\left(T^{*} L(T M) \otimes \mathbb{R}^{2 m}\right)$ the canonical 1-form,

- $h$ (resp. $h^{c}$ ) the horizontal lift associated with $\nabla$ (resp. $\left.\nabla^{c}\right)$,

- $G=\mathrm{GL}(m, \mathbb{R})$ and $P=L(M)$.

Theorem 3.1. Let $I$ be an open subset of $\mathbb{R}^{n},(X(a))_{a \in I}$ be a $C^{1}$-family of continuous semimartingales on $M$ with infinite lifetime, and let $\Theta_{0, .}(a)$ be the deformed parallel translation along $X(a)$. Let $a \mapsto U_{0}(a) \in P_{X_{0}(a)}$ be $C^{1}$ in probability, and let $U_{t}(a)=$ $\Theta_{0, t}(a) \circ U_{0}(a)$.

(i) Then $a \mapsto \Theta_{0, .}(a)$ is $C^{1}$ in the topology of semimartingales, and

$$
\Theta_{0, t}^{c}(a):=\left(\imath_{M} T U_{t}(a)\right) \circ\left(\imath_{M} T U_{0}(a)\right)^{-1}
$$

is the deformed parallel translation along $T X(a)$ with respect to $\nabla^{c}$.

(ii) If $Z(a)=\mathscr{A}_{\text {def }}(X(a))$ is the deformed antidevelopment of $X(a)$, then $a \mapsto Z(a)$ is $C^{1}$ in the topology of semimartingales, and for $v \in T I$, denoting by $\mathscr{A}_{\mathrm{def}}^{c}(T X(v))$ the deformed antidevelopment of $T X(v)$ with respect to the complete lift $\nabla^{c}$ of $\nabla$, we get

$$
\mathscr{A}_{\text {def }}^{c}(T X(v))=s_{M} T \mathscr{A}_{\text {def }}(X)(v) .
$$

(iii) Let $J=T X$; define $\nabla_{J} \Theta_{0,}: T_{X_{0}} N \rightarrow T_{X} . N$ by

$$
\left(\nabla_{J} \Theta_{0, .}\right) u=v_{J}^{-1}\left(\left(\Theta_{0, .}^{c} h_{J_{0}}(u)\right)^{\mathrm{vert}}\right),
$$


and $\nabla_{J} Z: T_{X_{0}} N \rightarrow T_{X_{0}} N$ by

$$
\left(\nabla_{J} Z\right) u=v_{J_{0}}^{-1}\left((s T Z(u))^{\mathrm{vert}}\right) .
$$

Then

$$
\nabla_{J} Z=\Theta_{0, .}^{-1} J-J_{0}-\int_{0} \Theta_{0, .}^{-1}\left(\nabla_{J} \Theta_{0, .}\right) d Z .
$$

Proof. We need the following relations which have been established in [2] Lemma 2.6:

$$
\begin{gathered}
\imath_{M}^{*} \omega^{c}=T \imath_{m} \circ s_{G} \circ T \omega \circ s_{P}, \\
\imath_{M}^{*} \vartheta^{c}=T \vartheta \circ s_{P}, \\
\imath_{M}^{-1} h^{c}=T \imath_{M} \circ s_{P} \circ T h \circ s_{M} .
\end{gathered}
$$

We are now ready to prove the theorem. For (i) and (ii), we may follow the proof of Theorem 3.1 in [2]; the difference is that Stratonovich differentials are replaced by Itô differentials, and the crucial result for differentiation with respect to a parameter is now Theorem 2.2.

(i) Let $U^{c}=\imath_{M} T U$. By Eq. (2.1), it is sufficient to show that $U^{c}$ is above $T X$ and $d^{\nabla^{c c}} U^{c}$ is horizontal or equivalently $\omega^{c}\left(d^{\nabla^{c c}} U^{c}\right)=0$. To see the first point we write

$$
\tilde{\pi} \circ U^{c}=\tilde{\pi} \circ \imath_{M} \circ T U=T(\pi \circ U)=T X .
$$

For the second point, using the Jacobi field characterization $\left(\nabla^{c}\right.$-geodesics are infinitesimal variations of $\nabla$-geodesics), it is first of all easy to check that $\imath_{M}$ sends geodesics in $T L(M)$ to geodesics in $L(T M)$, if both manifolds are endowed with the complete lift of the complete lift of the connection on $M$, which is denoted indifferently by $\nabla^{c c}$. As a consequence, we have

$$
d^{\nabla^{c c}} U^{c}=T \imath_{M} d^{\nabla^{c c}} T U
$$

Since $\omega\left(d^{\nabla^{c}} U\right)=0$, we get by differentiation along with Theorem 2.2 (which formally yields $T d^{\nabla^{c}} U=s_{P} \circ d^{\nabla^{c c}} T U$ )

$$
0=T \omega \circ s_{P} \circ\left(d^{\nabla^{c c}} T U\right)
$$

and thus by Eqs. (3.4) and (3.1)

$$
\omega^{c}\left(d^{\nabla^{c c}} U^{c}\right)=\omega^{c}\left(T \imath_{M} d^{\nabla^{c c}} T U\right)=T \imath_{m} \circ s_{G} \circ T \omega \circ s_{P} \circ\left(d^{\nabla^{c c}} T U\right)=0 .
$$

(ii) With the canonical projection $\pi:\left(L\left(T_{X_{0}} M, T M\right), \nabla^{c}\right) \rightarrow(M, \nabla)$ being affine, we get $\pi_{*} d^{\nabla^{c}} U(a)=d^{\nabla} X(a)$. This gives

$$
\int \vartheta\left(d^{\nabla^{c}} U(a)\right)=\int U(a)^{-1} \pi_{*} d^{\nabla^{c}} U(a)=\int U(a)^{-1} d^{\nabla} X(a) .
$$

As a consequence, $\bar{Z}(a):=\int \vartheta\left(d^{\nabla^{c}} U(a)\right)$ is the deformed antidevelopment of $X(a)$ into $\mathbb{R}^{m}$ with initial frame $U_{0}(a)$. Since by definition of $\imath_{M}$

$$
U_{0}^{c} T \bar{Z} \equiv \imath_{M}\left(T U_{0}\right)(T \bar{Z})=s_{M} T\left(U_{0} \bar{Z}\right),
$$

it suffices to verify that

$$
(T \bar{Z}) v=\bar{Z}^{c}(v),
$$


where $\bar{Z}^{c}(v)=\int \vartheta^{c}\left(d^{\nabla^{c c}} U^{c}(v)\right)$. But successively Theorem 2.2, (3.2) and (3.4) yield

$$
T\left(\vartheta d^{\nabla^{c}} U\right)=\left(T \vartheta \circ s_{P}\right) d^{\nabla^{c c}} T U=\left(\vartheta^{c} \circ T \imath_{M}\right) d^{\nabla^{c c}} T U=\vartheta^{c} d^{\nabla^{c c}} U^{c}
$$

which concludes the proof.

(iii) We know from [1] or Eq. (5.7) in Proposition 5.3 below

$$
d\left(\Theta_{0, .}^{-1} J\right)=\Theta_{0, .}^{-1}\left(v_{J}^{-1}\left(d^{\nabla^{c}} J\right)^{\text {vert }}\right) .
$$

By Theorem 2.2 we have in local coordinates

$$
\left(\begin{array}{c}
\left(d^{\nabla} X\right)^{i} \\
\left(d^{\left.\nabla^{c} J\right)^{i}}\right.
\end{array}\right)=\left(\begin{array}{cc}
\Theta_{\alpha}^{i} & 0 \\
T \Theta_{\alpha}^{i} & \Theta_{\alpha}^{i}
\end{array}\right)\left(\begin{array}{c}
d Z^{\alpha} \\
(d T Z)^{\alpha}
\end{array}\right)
$$

On the other hand, (ii) yields

$$
d^{\nabla^{c}} J=\Theta_{0, .}^{c} d(s T Z)
$$

Splitting $d(s T Z)$ into its horizontal and vertical part and then using the expression for $\Theta_{0, .}^{c}$ on vertical vectors given in (3.6), we derive from (3.7)

$$
\begin{aligned}
d^{\nabla^{c} J} & =\Theta_{0, .}^{c}\left(h_{J_{0}}(d Z)+v_{J_{0}}\left(d \nabla_{J} Z\right)\right) \\
& =\Theta_{0, .}^{c} h_{J_{0}}(d Z)+v_{J} \Theta_{0,} . d \nabla_{J} Z .
\end{aligned}
$$

The vertical part of the last equality gives

$$
v_{J}^{-1}\left(d^{\nabla^{c}} J\right)^{\mathrm{vert}}=\nabla_{J} \Theta_{0, .} d Z+\Theta_{0, .} d \nabla_{J} Z,
$$

thus along with Eq. (3.5) we get

$$
d \nabla_{J} Z=d\left(\Theta_{0, .}^{-1} J\right)-\Theta_{0, .}^{-1}\left(\nabla_{J} \Theta_{0, .}\right) d Z .
$$

Integrating this equation finally gives

$$
\nabla_{J} Z=\Theta_{0, .}^{-1} J-J_{0}-\int_{0} \Theta_{0, .}^{-1}\left(\nabla_{J} \Theta_{0, .}\right) d Z
$$

which is the desired relation.

\section{Horizontal Lifts to Vector Bundles}

Let $M$ be a manifold equipped with a connection $\nabla$ and let $\pi: E \rightarrow M$ be a vector bundle over $M$ equipped with a covariant derivative $\nabla^{E}$. We denote by $R$ (resp. $R^{E}$ ) the curvature tensor with respect to $\nabla$ (resp. $\nabla^{E}$ ). The tangent bundle $T E$ splits into $H E \oplus V E$ where $V E$ is the vertical bundle and $T_{e} \pi \mid H_{e} E$ is an isomorphism onto $T_{\pi(e)} M$ for every $e \in E$. Let $h_{e}=\left(T_{e} \pi \mid H_{e} E\right)^{-1}: T_{\pi(e)} M \rightarrow H_{e} E$ be the "horizontal lift", and let $v_{e}$ be the vertical lift $E_{\pi(e)} \rightarrow V_{e} E$. Every section $s \in \Gamma(E)$ has a canonical vertical lift $s^{v} \in \Gamma(T E)$ defined by $s_{e}^{v}=v_{e}\left(s_{\pi(e)}\right)$, and every vector field $X \in \Gamma(T M)$ a horizontal lift $X^{h} \in \Gamma(T E)$ defined by $X_{e}^{h}=h_{e}\left(X_{\pi(e)}\right)$.

The following result is well-known ([11]):

Proposition 4.1. There exists a unique connection $\nabla^{h}$ on $E$, i.e., a covariant derivative on TE,

$$
\nabla^{h}: \Gamma(T E) \rightarrow \Gamma\left(T^{*} E \otimes T E\right)
$$


satisfying the following properties: for all sections $r, s \in \Gamma(E), X, Y \in \Gamma(T M)$,

(4.1) $\quad \nabla_{r^{v}}^{h} s^{v}=0, \quad \nabla_{r^{v}}^{h} Y^{h}=0, \quad \nabla_{X^{h}}^{h} s^{v}=\left(\nabla_{X}^{E} s\right)^{v}, \quad \nabla_{X^{h}}^{h} Y^{h}=\left(\nabla_{X} Y\right)^{h}$.

Proof. The uniqueness is obvious: if $t \mapsto x(t) \in M, t \mapsto r(t) \in E_{x(t)}, t \mapsto f_{\ell}(t) \in \mathbb{R}$, $t \mapsto X_{\ell}(t) \in T_{x(t)} M, t \mapsto s_{\ell}(t) \in E_{x(t)}, 1 \leq \ell \leq r$, are smooth paths defined on $\mathbb{R}$, then

$$
\nabla_{D}^{h}\left(\sum_{\ell=1}^{r} f_{\ell}(t) X_{\ell}^{h}(t)\right)=\sum_{\ell=1}^{r}\left(f_{\ell}^{\prime}(t) h_{r(t)}\left(X_{\ell}(t)\right)+f_{\ell}(t) h_{r(t)}\left(\left(\nabla_{D} X_{\ell}\right)(t)\right)\right)
$$

and

$$
\nabla_{D}^{h}\left(\sum_{\ell=1}^{r} f_{\ell}(t) s_{\ell}^{v}(t)\right)=\sum_{\ell=1}^{r}\left(f_{\ell}^{\prime}(t) v_{r(t)}\left(s_{\ell}(t)\right)+f_{\ell}(t) v_{r(t)}\left(\left(\nabla_{D}^{E} s_{\ell}\right)(t)\right)\right) .
$$

For the existence, we have to verify that if for all $t$

$$
\sum_{\ell=1}^{r} f_{\ell}(t) X_{\ell}(t)=0 \quad \text { and } \quad \sum_{\ell=1}^{r} f_{\ell}(t) s_{\ell}(t)=0
$$

then

$$
\sum_{\ell=1}^{r}\left(f_{\ell}^{\prime}(0) h_{r(0)}\left(X_{\ell}(0)\right)+f_{\ell}(0) h_{r(0)}\left(\left(\nabla_{D} X_{\ell}\right)(0)\right)\right)=0
$$

and

$$
\sum_{\ell=1}^{r}\left(f_{\ell}^{\prime}(0) v_{r(0)}\left(s_{\ell}(0)\right)+f_{\ell}(0) v_{r(0)}\left(\left(\nabla_{D}^{E} s_{\ell}\right)(0)\right)\right)=0 .
$$

But the left hand side of (4.2) is equal to

$$
h_{r(0)}\left(\nabla_{D}\left(\sum_{\ell=1}^{r} f_{\ell} X_{\ell}\right)(0)\right)
$$

and the left hand side of (4.3) is equal to

$$
v_{r(0)}\left(\nabla_{D}^{E}\left(\sum_{\ell=1}^{r} f_{\ell} s_{\ell}\right)(0)\right)
$$

hence both terms vanish.

The connection $\nabla^{h}$ will be called the horizontal lift of $\left(\nabla, \nabla^{E}\right)$ to $E$, or simply the horizontal lift of $\nabla$ if there is a canonical way of deducing $\nabla^{E}$ from $\nabla$.

Let $S$ be a $E$-valued semimartingale. The parallel transport $/ /_{0, t}^{h} V$ of a vector $V=$ $X^{h}+r^{v}$ along $S_{t}$ with respect to $\nabla^{h}$ is given by

$$
/ /_{0, t}^{h} V=h_{S_{t}}\left(/ /_{0, t} X\right)+v_{S_{t}}\left(/ /_{0, t}^{E} r\right)
$$

where $/ /_{0, t}$ is parallel translation along $\pi\left(S_{t}\right)$ w.r.t. $\nabla$ and $/ /_{0, t}^{E}$ is parallel translation along $\pi\left(S_{t}\right)$ w.r.t. $\nabla^{E}$.

Similarly to [2], Theorem 4.2 and Corollary 4.4 , we get 
Theorem 4.2. Let $J$ be a continuous E-valued semimartingale and $X=\pi \circ J$.

1) The antidevelopment of $J$ with respect to $\nabla^{h}$ is given by the formula

$$
\mathscr{A}^{h}(J)=h_{J_{0}}(\mathscr{A}(X))+v_{J_{0}}\left(/ /_{0, .}^{E-1} J-J_{0}\right) .
$$

2) The semimartingale $J$ is a $\nabla^{h}$-martingale if and only if

(i) $X=\pi \circ J$ is a $\nabla$-martingale in $M$, and

(ii) $\left(/ / /_{0, t}^{E}\right)^{-1} J_{t}$ is a local martingale in $E_{X_{0}}$.

In the special case $E=T M$ we may compare the horizontal lift $\nabla^{h}$, as defined in this section, with the complete lift $\nabla^{c}$ defined in Section 2. In explicit terms we get for $V, W \in T_{p} T M, p \in T M$,

$$
\begin{aligned}
& \left(\nabla^{c}-\nabla^{h}\right)(V, W) \\
& \quad=v_{p}\left(R\left(p, \pi_{*} V\right) \pi_{*} W+\nabla_{\pi_{*} V} T\left(p, \pi_{*} W\right)+T\left(v_{p}^{-1} V^{\mathrm{vert}}, \pi_{*} W\right)\right)
\end{aligned}
$$

where $R: \Gamma(T M \otimes T M) \rightarrow \Gamma(\operatorname{End}(T M))$, resp. $T: \Gamma(T M \otimes T M) \rightarrow \Gamma(T M)$, is the curvature (resp. torsion) tensor to the connection $\nabla$ on $M$ and $v_{p}: T_{\pi(p)} M \rightarrow$ $T_{p} T M$ the vertical lift. Eq. (4.6) yields the following formula relating the Itô differentials for a $T M$-valued semimartingale $J$ with projection $\pi(J)=X$ :

$$
d^{\nabla^{c}} J=d^{\nabla^{h}} J+\frac{1}{2} v_{J}(R(J, d X) d X+\nabla T(d X, J, d X)+T(D J, d X))
$$

where $D J=/ / 0, . d\left(/ / 0_{0, .}^{-1} J\right)=v_{J}^{-1}\left(\left(d^{\nabla^{h}} J\right)^{\text {vert }}\right)$ (see Eq. (4.8) below).

Remark 4.3. Covariant derivatives on vector bundles induce covariant derivatives on tensor products and wedge products in a straightforward way: the parallel transport obtained is the tensor product, respectively wedge product of the given transports. A covariant derivative is also canonically induced on dual bundles: the associated parallel transport is the dual of the inverse parallel transport in the vector bundle. All these operations yield horizontal connections on tensor and wedge products of vector bundles and on dual vector bundles.

In the remainder of this section we consider $C^{1}$ families of $E$-valued semimartingales $a \mapsto J(a)$. We want to find a commutation formula for covariant derivatives with respect to $a$ and $t$.

Let $\nabla^{h h}$ be the horizontal lift on $T E$ of the connection $\nabla^{h}$ on $E$. As we shall see, the second part of Theorem 4.2 yields a simple characterization of $T E$-valued $\nabla^{h h}$ martingales.

First of all define $\psi: T E \rightarrow E$ by $\psi(W)=v_{w}^{-1}\left(W^{\text {vert }}\right)$ for $W \in T_{w} E$, and let $\pi_{2}$ be the canonical projection $T E \rightarrow E$. Then, by Theorem 4.2, a $T E$-valued semimartingale $W$ is a $\nabla^{h h}$-martingale if and only if

(i) $\pi_{2}(W)$ is an $E$-valued $\nabla^{h}$-martingale, and

(ii) $\left(/ /_{0}^{h} .\right)^{-1} W$ is a local martingale.

From Eq. (4.4) we have

$$
\left(/ /_{0, t}^{h}\right)^{-1} W_{t}=h_{\pi_{2}\left(W_{0}\right)}\left(/ /_{0, t}^{-1}\left(\pi_{*} W_{t}\right)\right)+v_{\pi_{2}\left(W_{0}\right)}\left(\left(/ /_{0, t}^{E}\right)^{-1}\left(\psi\left(W_{t}\right)\right)\right)
$$

By Theorem 4.2, condition (i) above implies that $X=\left(\pi \circ \pi_{2}\right)(W)$ is a $\nabla$-martingale; hence again by Theorem 4.2 (used twice) conditions (i) and (ii) are equivalent to 
(i) $\pi_{2}(W)$ is an $E$-valued $\nabla^{h}$-martingale,

(ii) v $_{(W)}$ is an $E$-valued $\nabla^{h}$-martingale, and

(ii) ${ }_{\mathrm{h}} \pi_{*}(W)$ is a $T M$-valued $\nabla^{h}$-martingale,

where $\nabla^{h}$ in item (ii) $)_{\mathrm{h}}$ is the horizontal lift of $\nabla$ in $T M$. As a consequence, we notice that the maps $\pi_{2}:\left(T E, \nabla^{h h}\right) \rightarrow\left(E, \nabla^{h}\right), \psi:\left(T E, \nabla^{h h}\right) \rightarrow\left(E, \nabla^{h}\right)$ and $\pi_{*}:\left(T E, \nabla^{h h}\right) \rightarrow\left(T M, \nabla^{h}\right)$ are semi-affine, i.e. they are affine with respect to the symmetrized connections.

If $J$ is an $E$-valued semimartingale, we define the covariant (Itô) derivative of $J$ by $D J:=\psi\left(d^{\nabla^{h}} J\right)$. Equivalently, $D J$ is characterized by the formula

$$
d^{\nabla^{h} J}=h_{J}\left(d^{\nabla} X\right)+v_{J}(D J) .
$$

Note that Eq. (4.5) yields

$$
D J=/ /_{0, .}^{E} d\left(/_{0,}^{E}{ }^{-1} J\right) .
$$

In local coordinates, write $\nabla^{E}$ as $d+A$ and $\nabla$ as $d+\Gamma$. Since

$$
\delta / /_{0, .}^{E}=-A\left(\delta X, / /_{0, .}^{E}\right),
$$

we have

$$
\delta\left(/ /_{0, .}^{E}\right)^{-1}=\left(/ /_{0, .}^{E}\right)^{-1} A(\delta X, \cdot)
$$

and hence

$$
\begin{aligned}
d\left(/ / /_{0, .}^{E}\right)^{-1}=\left(/ /_{0, .}^{E}\right)^{-1} A(d X, \cdot) & +\frac{1}{2}\left(/ / /_{0, .}^{E}\right)^{-1} d A(d X, d X, \cdot) \\
& +\frac{1}{2}\left(/ / /_{0, .}^{E}\right)^{-1} A(d X, A(d X, \cdot)) .
\end{aligned}
$$

Thus by Eq. (4.9) we get the following general formula for $(D J)^{\alpha}$ :

$$
\begin{aligned}
(D J)^{\alpha}=d J^{\alpha} & +A^{\alpha}(d X, J)+A^{\alpha}(d X, d J) \\
& +\frac{1}{2}\left(d A^{\alpha}(d X, d X, J)+A^{\alpha}(d X, A(d X, J))\right) .
\end{aligned}
$$

Substituting $d X=d^{\nabla} X-\frac{1}{2} \Gamma(d X, d X)$ in the second term of the r.h.s. then gives

$$
\begin{aligned}
(D J)^{\alpha}=d J^{\alpha} & +A^{\alpha}\left(d^{\nabla} X, J\right)+A^{\alpha}(d X, d J) \\
& +\frac{1}{2}\left(d A^{\alpha}(d X, d X, J)+A^{\alpha}(d X, A(d X, J))-A^{\alpha}(\Gamma(d X, d X), J)\right) .
\end{aligned}
$$

Taking into account that $(D J)^{\alpha}-d J^{\alpha}-A^{\alpha}(d X, J)$ has finite variation, and replacing $d J$ by $D J-A(d X, J)$ in the third term on the right, Eq. (4.11) finally leads to

$$
(D J)^{\alpha}=d J^{\alpha}+A^{\alpha}\left(d^{\nabla} X, J\right)+A^{\alpha}(d X, D J)+\frac{1}{2}\left(\nabla A^{\alpha}\right)(d X, d X, J) .
$$

Notation 4.4. If $a \mapsto w(a) \in E$ is a $C^{1}$ path, we denote by $\nabla_{a} w$ its covariant derivative: $\nabla_{a} w=\psi\left(\partial_{a} w\right)=\frac{D w}{d a}$ where $\psi(W)=v_{w}^{-1}$ ( $\left.W^{\text {vert }}\right)$ if $W \in T_{w} E$. Slightly abusing the notation, we just write $\nabla_{a_{0}} w$ for $\left.\nabla_{a}\right|_{a=a_{0}} w$.

The following theorem describes how covariant derivatives with respect to $a$ and $t$ commute. We write $\nabla R^{E}\left(v_{1}, v_{2}, v_{3}\right)$ for $\nabla_{v_{1}} R^{E}\left(v_{2}, v_{3}\right)$. 
Theorem 4.5. Let I be an open interval in $\mathbb{R}$, and for $a \in I$ let $J(a)$ be a semimartingale with values in the vector bundle $E$. Assume that $a \mapsto J(a)$ is $C^{1}$ in the topology of semimartingales. Let $X(a)=\pi(J(a))$. Then

$$
\begin{aligned}
D \nabla_{a} J= & \nabla_{a} D J+R^{E}\left(d^{\nabla} X, \partial_{a} X\right) J \\
& +R^{E}\left(d X, \partial_{a} X\right) D J-\frac{1}{2} \nabla R^{E}\left(d X, \partial_{a} X, d X\right) J-\frac{1}{2} R^{E}\left(D \partial_{a} X, d X\right) J .
\end{aligned}
$$

Remark 4.6. The Stratonovich version of this equation is

$$
D^{S} \nabla_{a} J=\nabla_{a} D^{S} J+R^{E}\left(\delta X, \partial_{a} X\right) J
$$

where $D^{S} J=/ /_{0 .}^{E} \delta\left(/ /_{0 .}^{E}{ }^{-1} J\right)$. In the particular situation when $J$ is parallel transport along $X$, Eq. (4.13) is a consequence of formula (4.7.5) in [15]. One could prove Theorem 4.5 with a Stratonovich to Itô conversion, but we prefer here to give a proof entirely based on the commutation formula (4.14) below.

Proof. Let $\nabla^{h c}$ be the complete lift of $\nabla^{h}$ in $T E$. We shall prove that the wanted formula is a consequence of the relation

$$
d^{\nabla^{h c}} \partial J=s\left(\partial d^{\nabla^{h}} J\right)
$$

given by Theorem 2.2 (when applied to Itô integrals as special cases of stochastic differential equations) and of formula

$$
\begin{aligned}
d^{\nabla^{h h}} \partial J= & d^{\nabla^{h c}} \partial J \\
& -\frac{1}{2} V_{\partial J}\left(R^{h}(\partial J, d J) d J+\nabla^{h} T^{h}(d J, \partial J, d J)+T^{h}\left(D^{h} \partial J, d J\right)\right)
\end{aligned}
$$

which is a consequence of (4.7). Here and in the sequel of the proof, $\partial$ stands for $\partial_{a}$, $R^{h}$ (resp. $T^{h}$ ) is the curvature (resp. torsion) tensor with respect to $\nabla^{h}$, and $V$ the vertical lift from $T E$ to $T T E$. A straightforward calculation using (4.1) shows that if $\mathcal{A}_{i}, 1 \leq i \leq 3$ are vectors in $T_{J} T E$ satisfying $\mathcal{A}_{i}=h_{J}\left(v_{i}\right)+v_{J}\left(A_{i}\right)$, then

$$
\begin{gathered}
R^{h}\left(\mathcal{A}_{1}, \mathcal{A}_{2}\right) \mathcal{A}_{3}=h_{J}\left(R\left(v_{1}, v_{2}\right) v_{3}\right)+v_{J}\left(R^{E}\left(v_{1}, v_{2}\right) A_{3}\right), \\
T^{h}\left(\mathcal{A}_{1}, \mathcal{A}_{2}\right)=h_{J}\left(T\left(v_{1}, v_{2}\right)\right)+v_{J}\left(R^{E}\left(v_{1}, v_{2}\right) J\right)
\end{gathered}
$$

and

$$
\begin{aligned}
\nabla^{h} T^{h}\left(\mathcal{A}_{1}, \mathcal{A}_{2}, \mathcal{A}_{3}\right)= & h_{J}\left(\nabla T\left(v_{1}, v_{2}, v_{3}\right)\right) \\
& +v_{J}\left(\nabla_{v_{1}} R^{E}\left(v_{2}, v_{3}\right) J+R^{E}\left(v_{2}, v_{3}\right) A_{1}\right) .
\end{aligned}
$$

The relation between $d^{\nabla^{h h}} \partial J$ and $D \nabla_{a} J$ is obtained as follows: from $\nabla_{a} J=\psi(\partial J)$ and the fact that $\psi$ is semi-affine we get $d^{\nabla^{h}} \nabla_{a} J=\psi_{*} d^{\nabla^{h h}} \partial J$ which gives

$$
D \nabla_{a} J=\psi\left(\psi_{*} d^{\nabla^{h h}} \partial J\right)
$$

One easily verifies that

$$
\psi \circ \psi_{*} \circ V=\psi
$$


which allows using Eqs. (4.8) (4.16), (4.17) and (4.18) to calculate the contribution of the second part of the r.h.s. of (4.15) in the wanted formula. More precisely, this contribution is seen to be

$$
-R^{E}(\partial X, d X) D J-\frac{1}{2} \nabla R^{E}(d X, \partial X, d X) J-\frac{1}{2} R^{E}(D \partial X, d X) J,
$$

when taking into account for the last term in the right that $D^{h} \partial J$ projects onto $D \partial X$ :

$$
\begin{aligned}
D^{h} \partial J & =/ /_{0, .}^{h} d\left(\left(/ /_{0, .}^{h}\right)^{-1} \partial J\right) \\
& =/ /_{0, .}^{h} d\left(h_{J_{0}} / /_{0, .}^{-1} \partial X+v_{J_{0}}\left(/ /_{0, .}^{E}\right)^{-1} \nabla_{a} J\right) \\
& =/ /_{0, .}^{h}\left(h_{J_{0}} d / /_{0, .}^{-1} \partial X+v_{J_{0}} d\left(/ /_{0, .}^{E}\right)^{-1} \nabla_{a} J\right) \\
& =h_{J} / /_{0, .} d / /_{0, .}^{-1} \partial X+v_{J} / /_{0, .}^{E} d\left(/ /_{0, .}^{E}\right)^{-1} \nabla_{a} J \\
& =h_{J} D \partial X+v_{J} D \nabla_{a} J .
\end{aligned}
$$

Next we calculate $\psi \circ \psi_{*}\left(d^{\nabla^{h c}} \partial J\right)$. Eqs. (4.14) and (4.8) yield

$$
\psi \circ \psi_{*}\left(d^{\nabla^{h c}} \partial J\right)=\psi \circ \psi_{*} \circ s\left(\partial h_{J}\left(d^{\nabla} X\right)\right)+\psi \circ \psi_{*} \circ s\left(\partial v_{J}(D J)\right) .
$$

We are left to verify that

$$
\psi \circ \psi_{*} \circ s\left(\partial h_{J}\left(d^{\nabla} X\right)\right)=R^{E}\left(d^{\nabla} X, \partial X\right) J
$$

and

$$
\psi \circ \psi_{*} \circ s\left(\partial v_{J}(D J)\right)=\nabla_{a} D J
$$

which is easily performed in local coordinates. Summing up the right hand sides of Eqs. (4.23), (4.22) together with (4.21) yields the claimed formula.

In the next four examples we illustrate all terms in the formula of Theorem 4.5.

Example 4.7. (Variation of parallel translation by a change of connection [5])

Let $M$ be a Riemannian manifold endowed with the Levi-Civita connection $\nabla$. Assume that $a \mapsto \nabla^{E}(a)$ is a $C^{1}$ family of covariant derivatives on $E$ indexed by $a \in I$ where $I$ is an open interval in $\mathbb{R}$ containing 0 . Let $\nabla^{h}(a)$ be the horizontal lift of $\left(\nabla, \nabla^{E}(a)\right)$; let $\nabla^{E}=\nabla^{E}(0)$ and $\nabla^{h}=\nabla^{h}(0)$. Denote by $\partial_{0} \nabla^{E}$ the derivative of $a \mapsto \nabla^{E}(a)$ at $a=0$. By definition, $\partial_{0} \nabla^{E}$ is a section of $T^{*} M \otimes E^{*} \otimes E$. Now let $X$ be a Brownian motion on $M$ and $W(a)(w)$ be the parallel transport along $X$ (with respect to $\nabla^{E}(a)$ ) of a vector $w \in E_{X_{0}}$ independent of $a$. Finally denote by $D^{a}$ the Itô covariant derivative with respect to $\nabla^{E}(a)$ and by $D$ the Itô covariant derivative with respect to $\nabla^{E}$. Recall that in local coordinates, by Eq. (4.11), writing $\nabla^{E}$ as $d+A$,

$$
\begin{aligned}
& (D W(a))^{\alpha}=d W^{\alpha}(a)+A^{\alpha}\left(d^{\nabla} X, W(a)\right)+A^{\alpha}(d X, d W(a)) \\
& +\frac{1}{2}\left(d A^{\alpha}(d X, d X, W(a))+A^{\alpha}(d X, A(d X, W(a)))-A^{\alpha}(\Gamma(d X, d X), W(a))\right) .
\end{aligned}
$$

Since $W(a)$ is the parallel transport with respect to $\nabla^{E}(a)$, we have $D^{a} W(a)=0$, hence

$$
D W(a)=\left(D-D^{a}\right) W(a) .
$$


For brevity, we write $W$ for $W(0)$. Differentiating Eq. (4.24) with the help of (4.25) yields

$$
\begin{array}{r}
\partial_{0}(D W(a))^{\alpha}=-\partial_{0} A^{\alpha}\left(d^{\nabla} X, W\right)-\partial_{0} A^{\alpha}(d X, d W)+\frac{1}{2}\left(-d \partial_{0} A^{\alpha}(d X, d X, W)\right. \\
\left.-\partial_{0} A^{\alpha}(d X, A(d X, W))-A^{\alpha}\left(d X, \partial_{0} A(d X, W)\right)+\partial_{0} A^{\alpha}(\Gamma(d X, d X), W)\right) .
\end{array}
$$

But $D W=0$ implies that $d W^{\alpha}+A^{\alpha}\left(d^{\nabla} X, W\right)$ has finite variation, and in particular

$$
\partial_{0} A^{\alpha}(d X, d W)=-\partial_{0} A^{\alpha}(d X, A(d X, W)) .
$$

Hence Eq. (4.26) gives

$$
\begin{aligned}
& \partial_{0}(D W(a))^{\alpha}=-\partial_{0} A^{\alpha}\left(d^{\nabla} X, W\right)+\frac{1}{2}\left(-d \partial_{0} A^{\alpha}(d X, d X, W)\right. \\
& \left.+\partial_{0} A^{\alpha}(d X, A(d X, W))-A^{\alpha}\left(d X, \partial_{0} A(d X, W)\right)+\partial_{0} A^{\alpha}(\Gamma(d X, d X), W)\right) .
\end{aligned}
$$

Now we replace $\partial_{0} A^{\alpha}$ by $\left(\partial_{0} \nabla^{E}\right)^{\alpha}$ to get the following intrinsic formula, where $\nabla_{0} D W$ stands again for $\left.\nabla_{a}\right|_{a=0} D W(a)$ :

$$
\nabla_{0} D W=-\partial_{0} \nabla^{E}\left(d^{\nabla} X, W\right)-\frac{1}{2} \nabla \partial_{0} \nabla^{E}(d X, d X, W) .
$$

Since $X$ is a Brownian motion, Eq. (4.28) can be written as

$$
\nabla_{0} D W=-\partial_{0} \nabla^{E}\left(d^{\nabla} X, W\right)+\frac{1}{2} d^{*} \partial_{0} \nabla^{E}(W) d t
$$

where $d^{*} \partial_{0} \nabla^{E}(W)=-\operatorname{tr} \nabla \partial_{0} \nabla^{E}(\cdot, \cdot, W)$. The other terms in the r.h.s. of the formula in Theorem 4.5 vanish, hence

$$
D \nabla_{0} W=-\partial_{0} \nabla^{E}\left(d^{\nabla} X, W\right)+\frac{1}{2} d^{*} \partial_{0} \nabla^{E}(W) d t
$$

and since $\nabla_{0} W$ projects onto $X$, by (4.8) it is a $\nabla^{h}$-martingale if $\partial_{0} \nabla^{E} \in \operatorname{Ker} d^{*}$.

Eq. (4.30) along with (4.8) recovers the following equivalence ([5] Proposition 4.2): $\partial_{0} \nabla^{E} \in \operatorname{Ker} d^{*}$ if and only if, for every $X$ and $W$ as above, $\nabla_{0} W$ is a $\nabla^{h}$-martingale.

Example 4.8. (Variation of parallel transport induced by a perturbation of the Brownian motion along the flow of a vector field [5]) Here $M$ is a Riemannian manifold endowed with the metric $g$ and Levi-Civita connection $\nabla, X$ is an $M$-valued Brownian motion, $X(a)=\phi_{a}(X)$ where $\left(\phi_{a}\right)_{a \in I}$ is the flow to a vector field $A \in \Gamma(T M)$ parameterized by some open interval $I \subset \mathbb{R}$ about 0 . The connection $\nabla^{h}$ is fixed and defined as horizontal lift of $\left(\nabla, \nabla^{E}\right)$ where $\nabla^{E}$ is a covariant derivative on $E$. For $w(a) \in E_{X_{0}(a)}$, let $W(a)(w(a))$ be the parallel transport of $w(a)$ along $t \mapsto X_{t}(a)$, i.e.,

$$
W_{0}(a)(w(a))=w(a), \quad \pi(W(a)(w(a)))=X(a), \quad D W(a)(w(a))=0 .
$$

Let $J(a)=W(a)(w(a))$ where $a \mapsto w(a) \in E_{X_{0}(a)}$ is $C^{1}$. For simplicity, we write again $\nabla_{0} J$ for $\left.\nabla_{a}\right|_{a=0} J$ and $\partial_{0} X$ for $\left.\partial_{a}\right|_{a=0} X$. Theorem 4.5 applied at $a=0$ gives

$$
D \nabla_{0} J=R^{E}\left(d^{\nabla} X, \partial_{0} X\right) J-\frac{1}{2} \nabla R^{E}\left(d X, \partial_{0} X, d X\right) J-\frac{1}{2} R^{E}\left(D \partial_{0} X, d X\right) J .
$$


Now from $X(a)=\phi_{a}(X)$ we get $\partial_{a} X(a)=A_{X(a)}$ which in turn implies that $D \partial_{0} X-$ $\nabla_{d \nabla} A$ is of finite variation. In particular, since $X$ is a Brownian motion,

$$
D \partial_{0} X \wedge d X=\operatorname{tr}(\nabla \cdot A \wedge \cdot)(X) d t .
$$

On the other hand, denoting by $\sharp$ the canonical isometry $\wedge^{k} T^{*} M \rightarrow \wedge^{k} T M$ induced by the metric and by $b$ its inverse, a calculation shows that

$$
\left(d A^{b}\right)^{\sharp}=-\operatorname{tr}(\nabla \cdot A \wedge \cdot) .
$$

Consequently, Eq. (4.31) transforms to

$$
D \nabla_{0} J=R^{E}\left(d^{\nabla} X, A(X)\right) J+\frac{1}{2}\left(-d^{*} R^{E}(A(X)) J+R^{E}\left(\left(d A^{b}\right)^{\sharp}\right) J\right) d t
$$

where again $d^{*} R^{E}(u)=-\operatorname{tr} \nabla R^{E}(\cdot, \cdot, u)$ for $u \in T M$. Defining

$$
\left(\nabla_{a} W(a)\right) w(a)=\nabla_{a}(W(a)(w(a)))-W(a)\left(\nabla_{a} w(a)\right)
$$

and taking into account that $D W(a)=0$, Eq. (4.32) yields

$$
D \nabla_{0} W=R^{E}\left(d^{\nabla} X, A(X)\right) W+\frac{1}{2}\left(-d^{*} R^{E}(A(X)) W+R^{E}\left(\left(d A^{b}\right)^{\sharp}\right) W\right) d t .
$$

Since $\nabla_{a} W$ projects onto $X$ at $a=0$, it is a $\nabla^{h}$-martingale if and only if

$$
-\frac{1}{2} d^{*} R^{E}(A)+\frac{1}{2} R^{E}\left(\left(d A^{b}\right)^{\sharp}\right)=0 .
$$

Now assume that $A$ is of gradient type $\left(d A^{\mathrm{b}}=0\right)$. Then we get

$$
D \nabla_{0} W=R^{E}\left(d^{\nabla} X, A(X)\right) W-\frac{1}{2} d^{*} R^{E}(A(X)) W d t .
$$

Moreover Eq. (4.34) yields the following equivalence ([5] Theorem 4.4): $\nabla^{E}$ is YangMills $\left(d^{*} R^{E}=0\right)$ if and only if $\nabla_{0} W$ is a $\nabla^{h}$-martingale for every $W$ as above.

Example 4.9. (Covariant derivative of parallel transport and Yang-Mills connections) $M$ is now a Riemannian manifold, $\nabla$ is the Levi-Civita connection associated with the metric, $E$ is endowed with a metric which is preserved by $\nabla^{E}$. Suppose $X$ is an $M$ valued $\mathrm{BM}$ on $M$ with lifetime $\xi ; / / 0$,. is the parallel transport in $T M$ along $X$; for $u \in T_{X_{0}} M, a \mapsto X_{t}(a, u)$ is the geodesic starting from $X_{t}$ with speed $\sqrt{t} / /_{0, t} u$ at $a=0$, i.e., $X_{t}(a, u)=\exp _{X_{t}}\left(a \sqrt{t} / /_{0, t} u\right)$. Finally, let $W_{t}(a, u)(\cdot)$ denote parallel translation in $E$ along $X_{t}(a, u)$ :

$$
\pi\left(W_{t}(a, u)\right)=X_{t}(a, u), \quad D W(a, u)=0, \quad W_{0}(a, u)=\operatorname{id}_{E_{X_{0}}} .
$$

Note that $W_{t}(a, u)(\cdot)$ is an isometry under the assumptions above. In terms of the covariant derivative $\nabla_{0} W$ of $W$ we consider the following $L\left(T_{X_{0}}^{*} M \otimes \operatorname{End}\left(E_{X_{0}}\right)\right)$ valued semimartingale:

$$
\begin{aligned}
W^{-1} \nabla_{0} W: T_{X_{0}} M & \longrightarrow \operatorname{End}\left(E_{X_{0}}\right) \\
u & \longmapsto W^{-1} \nabla_{0} W(u) .
\end{aligned}
$$

Recall that $\left.\nabla_{0} \equiv \nabla_{a}\right|_{a=0}$, thus $\left.\left(W^{-1} \nabla_{0} W\right)_{t}(u) \equiv W_{t}^{-1}(0, u) \nabla_{a}\right|_{a=0} W_{t}(a, u)$ where $W_{t}(0, u)=/ /_{0, t}^{E}$. 
Proposition 4.10. The semimartingale $W^{-1} \nabla_{0} W$ has lifetime $\xi$ and satisfies the Itô equation

$$
d\left(W^{-1} \nabla_{0} W\right)_{t}=W_{t}^{-1} R^{E}\left(d^{\nabla} X_{t}, \sqrt{t} / /_{0, t}\right) W_{t}-\frac{1}{2} W_{t}^{-1} d^{*} R^{E}\left(\sqrt{t} / /_{0, t}\right) W_{t} d t .
$$

The Riemannian quadratic variation $S_{t}^{\mathrm{var}}$ of $W^{-1} \nabla_{0} W$ is given by

$$
S_{t}^{\mathrm{var}}=\int_{0}^{t} 2 s\left\|R^{E}\left(X_{s}\right)\right\|^{2} d s
$$

where the norm of $R^{E}$ is the Euclidean norm in $L\left(\wedge^{2} T M\right.$, End $\left.E\right)$.

The covariant derivative $\nabla^{E}$ is Yang-Mills if and only if, for every $W$ as above, $W^{-1} \nabla_{0} W$ is a local martingale.

Remark 4.11. 1) The factor 2 in the r.h.s. of Eq. (4.37) disappears if one takes Euclidean norm in $L\left(\otimes^{2} T M\right.$, End $\left.E\right)$ instead of Euclidean norm in $L\left(\wedge^{2} T M\right.$, End $\left.E\right)$.

2) The factor $\sqrt{t}$ in $X_{t}(a, u)=\exp _{X_{t}}\left(a \sqrt{t} / /_{0, t} u\right)$ is introduced merely for scaling reasons. For instance, in case $M=\mathbb{R}^{m}$ the perturbation is $X_{t}(a, u)=X_{t}+a \sqrt{t} u$, and the factor $\sqrt{t}$ ensures Brownian scaling of the perturbed process. Note that in Eq. (4.37) our scaling convention leads to the additional multiplicative factor $s$.

Proof of Proposition 4.10. Let $\xi(a, u)$ denote the lifetime of $X(a, u)$ which is also the lifetime of $W(a, u)$. Then $\xi \wedge \xi(a, u)$ converges almost surely to $\xi$ as $a$ tends to 0 . This implies that $\left(W^{-1} \nabla_{0} W\right)(u)$ has lifetime $\xi$ as well. To establish Eq. (4.36) we compute $D \nabla_{0} W$ by means of Theorem 4.5. The only difference to Example 4.8 is that now $D \partial_{0} X_{t}(\cdot, u)=D\left(\sqrt{t} / /_{0, t} u\right)=\frac{1}{2 \sqrt{t}} / /_{0, t} u d t$ is a term of finite variation, with the consequence that $R^{E}\left(D \partial_{0} X, d X\right) W$ vanishes. Thus we get

$$
D \nabla_{0} W_{t}=R^{E}\left(d^{\nabla} X_{t}, \sqrt{t} / /_{0, t}\right) W_{t}-\frac{1}{2} d^{*} R^{E}\left(\sqrt{t} / /_{0, t}\right) W_{t} d t .
$$

To obtain Eq. (4.36) it is sufficient to invoke (4.9) which gives

$$
W^{-1} D \nabla_{0} W=d\left(W^{-1} \nabla_{0} W\right) .
$$

Eq. (4.37) follows from Eq. (4.36) along with the fact that $/ / 0$, and $W$ are isometries and $X$ is a Brownian motion.

For the last assertion we may proceed as in the proof of [5] Theorem 4.4: if $\nabla^{E}$ is Yang-Mills, then the drift in Eq. (4.36) disappears and $W^{-1} \nabla_{0} W$ is a local martingale. Conversely, if for every $W$ as above $W^{-1} \nabla_{0} W$ is a local martingale, choosing a Brownian motion $X$ starting from $x \in M$, we obtain almost surely

$$
W_{t}^{-1} d^{*} R^{E}(\sqrt{t} / / 0, t) W_{t}=0 \text { for all } t<\xi .
$$

Finally, dividing in the left by $\sqrt{t}$ and then letting $t$ tend to 0 , we get $d^{*} R^{E}(x)=0$ as wanted. 
Example 4.12. (Asymptotics of the parallel transport along a rescaled Brownian bridge) Let $M$ be a smooth $m$-dimensional compact Riemannian manifold with its Levi-Civita connection $\nabla$ and $\pi: E \rightarrow M$ be a vector bundle over $M$ endowed with a connection $\nabla^{E}$. Let $x_{0} \in M, u \in T_{x_{0}} M, X(a)=X(a, u)$ satisfy $X_{0}(a)=\exp _{x_{0}}(a u)$ and for $t \in[0,1[$ :

$$
d^{\nabla} X_{t}(a)=a A\left(X_{t}(a)\right) d B_{t}+b_{t}(a) d t
$$

where $B$ is an $\mathbb{R}^{r}$-valued BM, $A \in \Gamma\left(\mathbb{R}^{r} \otimes T M\right)$ is such that for all $x \in M$, $A(x) A(x)^{*}=\operatorname{id}_{T_{x} M}$, and $\nabla A\left(x_{0}\right)=0$. Note that such a choice for $A$ is always possible, locally with an orthonormal frame whose covariant derivative vanishes at $x_{0}$, and globally with the help of a partition of unity. Finally the drift $b_{t}$ in (4.38) is given by

$$
b_{t}(a)=V_{t}\left(a, X_{t}(a)\right)
$$

where

$$
V_{t}(a, x)=a^{2} \operatorname{grad}_{x} \log p\left(a^{2}(1-t), x, x_{0}\right)
$$

and $p(t, x, \cdot)$ is the density at time $t$ of a BM when started at $x$ at time 0 . The process $X(a)$ is a rescaled Brownian bridge; more precisely $t \mapsto X_{t / a^{2}}(a)$ is a Brownian motion starting at $\exp _{x_{0}}(a u)$ and conditioned to be at $x_{0}$ at time $a^{2}$.

In the sequel we keep the notation $\partial_{0}$ for $\left.\partial_{a}\right|_{a=0}$ and $\nabla_{0}$ for $\left.\nabla_{a}\right|_{a=0}$. It is well known that

$$
b_{t}(0)=0 \quad \text { and } \quad \nabla_{0} b_{t}=-\frac{1}{1-t} \partial_{0} X .
$$

Differentiating (4.38) with respect to $a$ with the help of Theorem 2.2, taking the covariant derivative with Eqs. (4.7) and (4.8) gives (since $\nabla$ is torsion-free)

$$
D \partial_{a} X=a \nabla_{\partial_{a} X} A(X) d B+A(X) d B+\nabla_{a} b d t-\frac{1}{2} R\left(\partial_{a} X, d X\right) d X .
$$

At $a=0$, since $X_{0}(a)=\exp _{x_{0}}(a u)$ we get $\partial_{0} X_{0}=u$ and

$$
D \partial_{0} X=A\left(x_{0}\right) d B-\frac{\partial_{0} X}{1-t} d t
$$

hence $\partial_{0} X$ is a Brownian bridge in the Euclidean space $T_{x_{0}} M$, starting at $u$ and ending up at 0 at time 1. Note that in Eq. (4.43) the covariant differential $D \partial_{0} X$ is equal to $d \partial_{0} X$ since $X(0) \equiv x_{0}$.

Differentiating Eq. (4.42) at $a=0$ with the help of Theorem 4.5, since $X(0) \equiv x_{0}$, yields

$$
D \nabla_{0} \partial_{a} X=2 \nabla_{\partial_{0} X} A\left(x_{0}\right) d B+\nabla_{0} \nabla_{a} b d t .
$$

But $\nabla A\left(x_{0}\right)=0$, so Eq. (4.44) gives

$$
D \nabla_{0} \partial_{a} X=\nabla_{0} \nabla_{a} b d t .
$$

On the other hand, $a \mapsto X_{0}(a)=\exp _{x_{0}}(a u)$ is a geodesic, so $\nabla_{0} \partial_{a} X_{0}=0$. In fact a careful investigation of $b$ shows that

$$
\nabla_{0} \nabla_{a} b_{t} \equiv 0 \quad \text { and } \quad \nabla_{0} \partial_{a} X_{t} \equiv 0 .
$$


This will be proved in a forthcoming paper, together with the following theorem which describes the asymptotics in $a$ at $a=0$ (up to order three) of the parallel transport in $E$ along $X(a, u)$.

Theorem 4.13. Let $u \in T_{x_{0}} M$ and $W(a)=W(a, u)$ be the parallel transport in $E$ along $X(a, u)$. Derivatives with respect to a are taken in the topology of semimartingales. The following formulas hold:

$$
\begin{gathered}
W_{t}(0)=\mathrm{id}_{E_{x_{0}}}, \\
\nabla_{0} W_{t} \equiv 0, \\
\nabla_{0} \nabla_{a} W_{t}=\int_{0}^{t} R^{E}\left(A\left(x_{0}\right) d B_{s}, \partial_{0} X_{s}\right),
\end{gathered}
$$

and hence $\nabla_{0} \nabla_{a} W_{t}$ is a martingale,

$$
\nabla_{0} \nabla_{a} \nabla_{a} W_{t}=2 \int_{0}^{t} \nabla R^{E}\left(\partial_{0} X_{s}, A\left(x_{0}\right) d B_{s}, \partial_{0} X_{s}\right)-d^{*} R^{E}\left(\int_{0}^{t} \partial_{0} X_{s} d s\right) .
$$

We have the following asymptotic expansion at $a=0$

$$
\mathbb{E}\left[W_{1}(a) \tau_{0, a}\right]=\mathrm{id}_{E_{x_{0}}}-\frac{a^{3}}{12} d^{*} R^{E}(u)+\mathrm{O}\left(a^{4}\right)
$$

where $\tau_{0, a}$ is the parallel transport in $E$ along $a \mapsto \exp _{x_{0}}(a u)$. In particular, the following three assertions are equivalent:

(i) $d^{*} R^{E}$ vanishes at $x_{0}$

(ii) for every $u \in T_{x_{0}} M, \nabla_{0} \nabla_{a} \nabla_{a} W(a, u)$ is a martingale,

(iii) for every $u \in T_{x_{0}} M$,

$$
\mathbb{E}\left[W_{1}(a, u) \tau_{0, a}-\mathrm{id}_{E_{x_{0}}}\right]=\mathrm{O}\left(a^{4}\right) .
$$

Remark 4.14. 1) Eq. (4.43) shows that formula (4.49) can be rewritten as

$$
\nabla_{0} \nabla_{a} W_{t}=\int_{0}^{t} R^{E}\left(\delta \partial_{0} X_{s}, \partial_{0} X_{s}\right)
$$

which is identical to formula (39) in [4].

Similarly, Eq. (4.50) rewrites as the Stratonovich integral

$$
\nabla_{0} \nabla_{a} \nabla_{a} W_{t}=2 \int_{0}^{t} \nabla R^{E}\left(\partial_{0} X_{s}, \delta \partial_{0} X_{s}, \partial_{0} X_{s}\right)
$$

2) In [18] and [6] the authors obtained a condition similar to (4.52), but in their result the time is not fixed; it is the first exit time of a ball of radius $a\|u\|$. Here the full terms of the asymptotic expansion in $a$ are obtained, and the covariant derivative $\nabla^{E}$ is not required to be compatible with any metric. The proof is based on successive applications of Theorem 4.5 together with a careful investigation of the equations obtained. Finally to derive Eq. (4.52) which involves time 1 where the equations have a singularity, we use a time-reversal argument and the symmetry of the law of a Brownian bridge. 


\section{A General Class of Lifts to Vector Bundles}

Let $E$ be a vector bundle equipped with a horizontal connection $\nabla^{h}$. In this section we investigate connections $\nabla^{\mathscr{S}}$ on $E$ of the following type: for $A, B \in \Gamma(T E), J \in E$, let

$$
\nabla_{A_{J}}^{\mathscr{S}} B=\nabla_{A_{J}}^{h} B+\mathscr{S}\left(A_{J}, B_{J}\right)
$$

with

$$
\mathscr{S}\left(A_{J}, B_{J}\right)=v_{J}\left(\mathscr{R}\left(\pi_{*} A_{J}, \pi_{*} B_{J}\right) J+\mathscr{T}\left(v_{J}^{-1}\left(A^{\mathrm{vert}}\right), \pi_{*} B_{J}\right)\right)
$$

where $\mathscr{R}$ is a smooth section of $T^{*} M \otimes T^{*} M \otimes \operatorname{End}(E)$ over $M$ and $\mathscr{T}$ a smooth section of $E^{*} \otimes T^{*} M \otimes E$ over $E$ (and not over $M$ ).

Examples of such connections are given trivially by the horizontal lift itself, and the complete lift in the case $E=T M$. In the latter case we have

$$
\mathscr{R}(X, Y) J=R(J, X) Y+\left(\nabla_{X} T\right)(J, Y) \quad \text { and } \quad \mathscr{T}(X, Y)=T(X, Y)
$$

where $R$ and $T$ denote the curvature and the torsion tensor of $\nabla$ (here $T$ is considered as section of $T^{*} M \otimes T^{*} M \otimes T M$ over $M$ ). Other examples will be given in Sections 7 and 8.

Let us consider the three following properties for a connection $\nabla^{\prime}$ on $E$ :

(i) $\nabla^{\prime}-\nabla^{h}$ is vertical,

(ii) $\left(\nabla^{\prime}-\nabla^{h}\right)(\cdot, B)=0$ if $B$ is vertical (which implies that the flat vector space $E_{x}$ is affinely immersed in $\left(E, \nabla^{\prime}\right)$ for each $\left.x \in M\right)$.

(iii) $\left(\nabla^{\prime}-\nabla^{h}\right)\left(h_{J}(X), h_{J}(Y)\right)$ is linear in $J$ for fixed vector fields $X, Y \in \Gamma(T M)$.

The following result is immediate:

Proposition 5.1. A connection $\nabla^{\prime}$ on $E$ is of the form $\nabla^{\mathscr{S}}$ as defined by (5.1) for some $\mathscr{S}$ as in (5.2), if and only if $\nabla^{\prime}$ satisfies (i), (ii) and (iii).

Note that connections similar to $\nabla^{\mathscr{S}}$ but without torsion have already been studied in [16].

Let $\nabla^{\mathscr{S}}$ be a connection defined by (5.1) and (5.2). An immediate consequence of Eqs. (5.1) and (5.2) is that the Itô differential of an $E$-valued semimartingale $J$ satisfies

$$
d^{\nabla^{\mathscr{S}} J}=d^{\nabla^{h}} J+\frac{1}{2} v_{J}(\mathscr{R}(d X, d X) J+\mathscr{T}(D J, d X))
$$

where $X=\pi(J)$ and $D J_{t}=/ /_{0, t}^{E} d\left(\left(/ /_{0, t}^{E}\right)^{-1} J_{t}\right)=v_{J}^{-1}\left(\left(d^{\nabla^{h}} J\right)^{\text {vert }}\right)$.

If $X$ is an $M$-valued semimartingale we define the deformed parallel translation along $X$ as the semimartingale $\Theta_{0, t}$ with values in $\operatorname{Hom}\left(E_{X_{0}}, E_{X_{t}}\right)$, solution to

$$
\Theta_{0,0}=\operatorname{id}_{E_{X_{0}}}, \quad d^{\nabla^{\mathscr{S}}} \Theta_{0, t}=h_{\Theta_{0, t}}\left(d^{\nabla} X_{t}\right) .
$$

An equivalent definition to (5.5) is given by

$$
\Theta_{0,0}=\operatorname{id}_{E_{X_{0}}}, \quad \pi\left(\Theta_{0, t}\right)=X_{t}, \quad D \Theta_{0, t}=-\frac{1}{2} \mathscr{R}(d X, d X) \Theta_{0, t} .
$$

Note that the term $\mathscr{T}\left(D \Theta_{0, t}, d X_{t}\right)$ vanishes since $D \Theta_{0, .}$ is of finite variation. Also recall that $\Theta_{0 .}$ is defined via a linear equation and has consequently the same lifetime as $X$. 
Definition 5.2. A process $\Theta_{0,}$ as defined by Eq. (5.5) is called a horizontal $\nabla^{\mathscr{S}}$ semimartingale (or a $\nabla^{\mathscr{S}}$-transport in $E$ ). It is called a horizontal $\nabla^{\mathscr{S}}$-martingale if it projects to a $\nabla$-martingale $X$ on $M$.

As a generalization of Theorem 2.1, we get the following proposition. The second part has been proved in [16] for torsion-free connections:

Proposition 5.3. Let $J$ be a continuous semimartingale with values in $E$ and let $X=$ $\pi(J)$. Then

$$
d^{\nabla^{\mathscr{S}}} J_{t}=h_{J_{t}}\left(d^{\nabla} X_{t}\right)+v_{J_{t}}\left(\Theta_{0, t}\left(d\left(\Theta_{0, t}^{-1} J_{t}\right)\right)+\frac{1}{2} \mathscr{T}\left(D J_{t}, d X_{t}\right)\right) .
$$

Consequently, $J$ is a $\nabla^{\mathscr{S}}$-martingale if and only if

(i) $X=\pi(J)$ is a $\nabla$-martingale in $M$, and

(ii) $\Theta_{0, .}^{-1} J+\frac{1}{2} \int_{0} \Theta_{0, s}^{-1} \mathscr{T}\left(D J_{s}, d X_{s}\right)$ is a local martingale.

Proof. We only need to establish Eq. (5.7), the rest of the proof is an immediate consequence of it. Setting $\theta_{0, t}=\left(/ /\left.\right|_{0, t} ^{E}\right)^{-1} \Theta_{0, t}$ we have

$$
d \theta_{0, t}=\left(/ / /_{0, t}^{E}\right)^{-1} D \Theta_{0, t}=-\frac{1}{2}\left(/ /_{0, t}^{E}\right)^{-1} \mathscr{R}(d X, d X) \Theta_{0, t}
$$

which gives

$$
d \theta_{0, t}^{-1}=-\theta_{0, t}^{-1} d \theta_{0, t} \theta_{0, t}^{-1}=\frac{1}{2} \Theta_{0, t}^{-1} \mathscr{R}(d X, d X) / /_{0, t}^{E}
$$

We get

$$
\begin{aligned}
d\left(\Theta_{0, t}^{-1} J_{t}\right) & =d\left(\theta_{0, t}^{-1}\left(/ /_{0, t}^{E}\right)^{-1} J_{t}\right) \\
& =\left(d \theta_{0, t}^{-1}\right)\left(/ /_{0, t}^{E}\right)^{-1} J_{t}+\theta_{0, t}^{-1} d\left(\left(/ /_{0, t}^{E}\right)^{-1} J_{t}\right) \\
& =\frac{1}{2} \Theta_{0, t}^{-1} \mathscr{R}\left(d X_{t}, d X_{t}\right) J_{t}+\theta_{0, t}^{-1} d\left(\left(/ /_{0, t}^{E}\right)^{-1} J_{t}\right) \\
& =\Theta_{0, t}^{-1}\left(\frac{1}{2} \mathscr{R}\left(d X_{t}, d X_{t}\right) J_{t}+v_{J_{t}}^{-1}\left(\left(d^{\nabla^{h}} J_{t}\right)^{\text {vert }}\right)\right) \\
& =\Theta_{0, t}^{-1}\left(-\frac{1}{2} \mathscr{T}\left(D J_{t}, d X_{t}\right)+v_{J_{t}}^{-1}\left(\left(d^{\nabla^{\mathscr{S}}} J_{t}\right)^{\mathrm{vert}}\right)\right) .
\end{aligned}
$$

As a consequence, since $d^{\nabla^{h}} J$ and $d^{\nabla^{\mathscr{S}}} J$ have the same horizontal parts, we get similarly to Eq. (4.8)

$$
d^{\nabla^{\mathscr{S}}} J_{t}=h_{J_{t}}\left(d^{\nabla} X_{t}\right)+v_{J_{t}}\left(\Theta_{0, t}\left(d\left(\Theta_{0, t}^{-1} J_{t}\right)\right)+\frac{1}{2} \mathscr{T}\left(D J_{t}, d X_{t}\right)\right)
$$

which is the wanted result.

Remark 5.4. In most of the considered examples the term $\mathscr{T}$ vanishes, then condition (ii) in Proposition 5.3 simplifies. In some situations we confine ourselves by assumption to connections on $E$ with $\mathscr{T}=0$, in order to make operations on the lifts more natural, but then (for connections with torsion) our set-up does no longer generalize the notion 
of complete lift in tangent spaces, as defined by Eq. (5.3). In these cases however, the considered semimartingales $J$ will satisfy $D J \otimes d X=0$, which implies that we have nevertheless a consistent generalization as long as one stays in this class of processes.

We continue the investigation of $\nabla^{\mathscr{S}}$ by establishing formulas for the stochastic parallel transport and the antidevelopment. If $J$ is an $E$-valued semimartingale, let $D^{S} J_{t}=v_{J_{t}}^{-1}\left((\delta J)^{\mathrm{vert}}\right)=/ /_{0, t}^{E} \delta\left(\left(/ /_{0, t}^{E}\right)^{-1} J_{t}\right)$ be the vertical part of its Stratonovich differential, $/ /_{0, t}^{\mathscr{S}}$ the parallel transport along $J$ with respect to $\nabla^{\mathscr{S}}$, and $\mathscr{A}^{\mathscr{S}}(J)$ (resp. $\mathscr{A}^{h}(J)$ ) the antidevelopment of $J$ with respect to $\nabla^{\mathscr{S}}$ (resp. $\nabla^{h}$ ).

Proposition 5.5. Let $J$ be an E-valued semimartingale.

(1) The parallel transport $/ /_{0, t}^{\mathscr{S}}$ along $J$ is given as follows: For $w \in T_{J_{0}} E$ with projection $u \in T_{X_{0}} M$, we have

$/ /_{0, t}^{\mathscr{S}} w=/ /_{0, t}^{h} w-v_{J_{t}}\left(/ /_{0, t}^{E} \int_{0}^{t}\left(/ /_{0, s}^{E}\right)^{-1}\left(\mathscr{T}\left(D^{S} J_{s}, / /_{0, s} u\right)+\mathscr{R}\left(\delta X_{s}, / /_{0, s} u\right) J_{s}\right)\right)$.

(2) The antidevelopment $\mathscr{A}^{\mathscr{S}}(J)$ of $J$ satisfies

$$
\begin{aligned}
\mathscr{A}^{\mathscr{S}}(J)_{t} & =\mathscr{A}^{h}(J)_{t} \\
\quad+v_{J_{0}} & \left(\int_{0}^{t}\left(\int_{0}^{s}\left(/ /_{0, r}^{E}\right)^{-1}\left(\mathscr{T}\left(D^{S} J_{r}, / /_{0, r} \cdot\right)+\mathscr{R}\left(\delta X_{r}, / /_{0, r} \cdot\right) J_{r}\right)\right) \delta \mathscr{A}(X)_{s}\right) .
\end{aligned}
$$

Proof. (1) We may assume that $J$ is a smooth deterministic path and replace Stratonovich differentials by ordinary differentials. Let $w_{t}$ denote the r.h.s. of (5.8), and let $u_{t}=$ $/ / 0, t$. Since $\nabla^{\mathscr{S}}$ and $\nabla^{h}$ coincide on vertical vector fields, we have

$$
\nabla_{D}^{\mathscr{S}} / /_{0, t}^{h} w^{\mathrm{vert}}=0
$$

As a consequence, we get

$$
\begin{aligned}
& \nabla_{D}^{\mathscr{S}} w_{t} \\
& =\nabla_{D}^{\mathscr{S}}\left(/ / /_{0, t}^{h} w^{\mathrm{hor}}-v_{J_{t}}\left(/ /_{0, t}^{E} \int_{0}^{t}\left(/ /_{0, s}^{E}\right)^{-1}\left(\mathscr{T}\left(\nabla_{D} J_{s}, u_{s}\right)+\mathscr{R}\left(\dot{X}_{s}, u_{s}\right) J_{s}\right) d s\right)\right) .
\end{aligned}
$$

But Eqs. (4.4), (5.1) and (5.2) yield

$$
\nabla_{D}^{\mathscr{S}} / /_{0, t}^{h} w^{\text {hor }}=v_{J_{t}}\left(\mathscr{T}\left(\nabla_{D} J_{t}, u_{t}\right)+\mathscr{R}\left(\dot{X}_{t}, u_{t}\right) J_{t}\right)
$$

and from (4.4) and the fact that $\nabla^{\mathscr{S}}$ and $\nabla^{h}$ coincide on vertical vectors we get

$$
\begin{aligned}
\nabla_{D}^{\mathscr{S}} v_{J_{t}} & \left(/ / /_{0, t}^{E} \int_{0}^{t}\left(/ /\left.\right|_{0, s} ^{E}\right)^{-1}\left(\mathscr{T}\left(\nabla_{D} J_{s}, u_{s}\right)+\mathscr{R}\left(\dot{X}_{s}, u_{s}\right) J_{s}\right) d s\right) \\
& =v_{J_{t}}\left(/ / /_{0, t}^{E} \frac{d}{d t} \int_{0}^{t}\left(/ / /_{0, s}^{E}\right)^{-1}\left(\mathscr{T}\left(\nabla_{D} J_{s}, u_{s}\right)+\mathscr{R}\left(\dot{X}_{s}, u_{s}\right) J_{s}\right) d s\right) \\
& =v_{J_{t}}\left(\mathscr{T}\left(\nabla_{D} J_{t}, u_{t}\right)+\mathscr{R}\left(\dot{X}_{t}, u_{t}\right) J_{t}\right) .
\end{aligned}
$$

Thus $\nabla_{D}^{\mathscr{S}} w_{t}=0$ which gives the result. 
(2) We first calculate the inverse parallel transport with respect to $\nabla^{\mathscr{S}}$. Letting $w^{\prime}=/ /_{0, t}^{\mathscr{S}} w$, we have

$$
/ / h_{0, t}^{h} w=w^{\prime}+v_{J_{t}}\left(/ / /_{0, t}^{E} \int_{0}^{t}\left(/ /_{0, s}^{E}\right)^{-1}\left(\mathscr{T}\left(D^{S} J_{s}, / /_{0, s} u\right)+\mathscr{R}\left(\delta X, / /_{0, s} u\right) J_{s}\right)\right) .
$$

Applying $\left(/ /_{0, t}^{h}\right)^{-1}$ on both sides and using formula (4.4) yields with $u^{\prime}=\pi_{*} w^{\prime}$,

$$
\begin{aligned}
\left(/ / /_{0, t}^{\mathscr{S}}\right)^{-1} w^{\prime} & =\left(/ /_{0, t}^{h}\right)^{-1} w^{\prime} \\
& +v_{J_{0}}\left(\int_{0}^{t}\left(/ /_{0, s}^{E}\right)^{-1}\left(\mathscr{T}\left(D^{S} J_{s}, / /_{0, s} / /_{0, t}^{-1} u^{\prime}\right)+\mathscr{R}\left(\delta X_{s}, / /_{0, s} / /_{0, t}^{-1} u^{\prime}\right) J_{s}\right)\right) .
\end{aligned}
$$

Now from the formulas

$$
\delta \mathscr{A}^{h}(J)_{t}=\left(/ /_{0, t}^{h}\right)^{-1} \delta J_{t} \quad \text { and } \quad \delta \mathscr{A}^{\mathscr{S}}(J)_{t}=\left(/ /_{0, t}^{\mathscr{S}}\right)^{-1} \delta J_{t},
$$

we get

$$
\begin{aligned}
\delta & \left.\mathscr{A}^{\mathscr{S}}(J)_{t}-\mathscr{A}^{h}(J)_{t}\right) \\
& =\left(\left(/ /_{0, t}^{\mathscr{S}}\right)^{-1}-\left(/ /_{0, t}^{h}\right)^{-1}\right) \delta J_{t} \\
& =v_{J_{0}}\left(\int_{0}^{t}\left(/ /_{0, s}^{E}\right)^{-1}\left(\mathscr{T}\left(D^{S} J_{s}, / /_{0, s} / /_{0, t}^{-1} \pi_{*} \delta J_{t}\right)+\mathscr{R}\left(\delta X_{s}, / /_{0, s} / /_{0, t}^{-1} \pi_{*} \delta J_{t}\right) J_{s}\right)\right) \\
& =v_{J_{0}}\left(\left(\int_{0}^{t}\left(/ /_{0, s}^{E}\right)^{-1}\left(\mathscr{T}\left(D^{S} J_{s}, / /_{0, s} \cdot\right)+\mathscr{R}\left(\delta X_{s}, / /_{0, s} \cdot\right) J_{s}\right)\right) \delta \mathscr{A}(X)_{t}\right) .
\end{aligned}
$$

The proof is achieved by integrating the last formula.

For the remainder of this section we consider connections $\nabla^{\mathscr{S}}$ with vanishing $\mathscr{T}$ and construct lifts of connections on dual bundles and tensor bundles.

To a given section $\mathscr{R}^{E}$ of $T^{*} M \otimes T^{*} M \otimes$ End $E$, we first define a section $\mathscr{R}^{E^{*}}$ of $T^{*} M \otimes T^{*} M \otimes$ End $E^{*}$ by means of the formula

$$
\left\langle\mathscr{R}^{E^{*}}(X, Y) \alpha, J\right\rangle+\left\langle\alpha, \mathscr{R}^{E}(X, Y) J\right\rangle=0, \quad X, Y \in T_{x} M, J \in E_{x}, \alpha \in E_{x}^{*},
$$

thus $\mathscr{R}^{E^{*}}(X, Y)=-\left(\mathscr{R}^{E}(X, Y)\right)^{*}$. Now assume that we have two vector bundles $E$ and $F$ over the same base $M$ (for different base manifolds, say $M$ and $N$, first extend everything canonically to $M \times N)$. In addition, assume that sections $\mathscr{R}^{E}$ of $T^{*} M \otimes T^{*} M \otimes$ End $E^{*}$ and $\mathscr{R}^{F}$ of $T^{*} M \otimes T^{*} M \otimes$ End $F^{*}$ are given. We define a section $\mathscr{R}^{E \otimes F}$ of $T^{*} M \otimes T^{*} M \otimes \operatorname{End}(E \otimes F)$ by linearly extending the formula

$$
\mathscr{R}^{E \otimes F}(X, Y)\left(J_{1} \otimes J_{2}\right)=\left(\mathscr{R}^{E}(X, Y) J_{1}\right) \otimes J_{2}+J_{1} \otimes\left(\mathscr{R}^{F}(X, Y) J_{2}\right),
$$

where $X, Y \in T_{x} M, J_{1} \in E_{x}, J_{2} \in F_{x}$.

These formulas define connections on $E^{*}$, resp. $E \otimes F$, for simplicity again denoted by $\nabla^{\mathscr{S}}$, called the dual of the connection on $E$, resp. the tensor product of the connections on $E$ and $F$. Finally, for an $M$-valued semimartingale $X$, let $\Theta^{E}, \Theta^{E^{*}}$, resp. $\Theta^{E \otimes F}$, denote the deformed parallel translations along $X$ on $E, E^{*}$, resp. $E \otimes F$.

Proposition 5.6. (1) $\Theta^{E^{*}}=\left(\left(\Theta^{E}\right)^{-1}\right)^{*} \quad$ (2) $\Theta^{E \otimes F}=\Theta^{E} \otimes \Theta^{F}$. 
This result is similar to the results in [16] Sections 5 and 6, but the assumptions are not the same and the proof is different.

Proof. (1) It is sufficient to prove that

$$
D\left(\left(\Theta^{E}\right)^{-1}\right)^{*}=-\frac{1}{2} \mathscr{R}^{E^{*}}(d X, d X)\left(\left(\Theta^{E}\right)^{-1}\right)^{*} .
$$

But Remark 4.3 and formula (5.6) for the covariant derivative of $\Theta^{E}$ give

$$
\begin{aligned}
D\left(\left(\Theta^{E}\right)^{-1}\right)^{*} & =\left(/ /_{0, .}^{E-1}\right)^{*} d\left(\left(\left(/ /_{0, .}^{E-1}\right)^{*}\right)^{-1}\left(\left(\Theta^{E}\right)^{-1}\right)^{*}\right) \\
& =\left(/ /_{0, .}^{E-1}\right)^{*} d\left(\left(/ /_{0, .}^{E}\right)^{*}\left(\left(\Theta^{E}\right)^{-1}\right)^{*}\right) \\
& =\left(/ /_{0, .}^{E-1}\right)^{*} d\left(\left(\Theta^{E}\right)^{-1} / /_{0, .}^{E}\right)^{*} \\
& =\frac{1}{2}\left(/ / /_{0, .}^{E-1}\right)^{*}\left(\left(\Theta^{E}\right)^{-1} \mathscr{R}^{E}(d X, d X) / /_{0, .}^{E}\right)^{*} \\
& =\frac{1}{2}\left(/ /_{0, .}^{E-1}\right)^{*}\left(/ / /_{0, .}^{E}\right)^{*}\left(\mathscr{R}^{E}(d X, d X)\right)^{*}\left(\left(\Theta^{E}\right)^{-1}\right)^{*} \\
& =-\frac{1}{2} \mathscr{R}^{E^{*}}(d X, d X)\left(\left(\Theta^{E}\right)^{-1}\right)^{*}
\end{aligned}
$$

which is the desired relation.

(2) The result immediately follows from the relation

$$
D\left(\Theta^{E} \otimes \Theta^{F}\right)=D \Theta^{E} \otimes \Theta^{F}+\Theta^{E} \otimes D \Theta^{F}
$$

which is established by means of Remark 4.3. There is no bracket since by Eq. (5.6), $\left(/ /{ }_{0, .}^{E}\right)^{-1} \Theta^{E}$ is of finite variation.

If there is no danger of confusion, we denote the sections $\mathscr{R}^{E}, \mathscr{R}^{E^{*}}$ and $\mathscr{R}^{E \otimes F}$ without differentiation by the same symbol $\mathscr{R}$.

For a Riemannian manifold $M$, let $\mathscr{R}=\mathscr{R}^{E^{*}}$ be the section of $T^{*} M \otimes T^{*} M \otimes$ $\operatorname{End}\left(E^{*}\right)$ defined in (5.9). Setting $\operatorname{tr} \mathscr{R}=\sum_{i=1}^{n} \mathscr{R}\left(e_{i}, e_{i}\right)$, where $\left(e_{i}\right)_{1 \leq i \leq m}$ is a local section of the orthonormal frame bundle over $M$, we define the $\mathscr{R}$-Laplacian as

$$
\Delta^{\mathscr{R}}:=\square+\operatorname{tr} \mathscr{R},
$$

where $\square:=\operatorname{tr}\left(\nabla^{T^{*} M \otimes E^{*}} \circ \nabla^{E^{*}}\right)$ is the horizontal Laplacian on forms.

Let $\Theta_{0, .}$ denote the $\nabla^{\mathscr{S}}$-transport on $E$ along a semimartingale $X$. We continue to assume that $\mathscr{T}$ vanishes.

Proposition 5.7. Assume that $(M, \nabla)$ is a Riemannian manifold equipped with the LeviCivita connection and let $X$ be an $M$-valued Brownian motion. For every e $\in E_{X_{0}}$, the restriction of the generator of $/ /_{0, t}^{E}$ e (resp. $\Theta_{0, t}$ e) to sections of $E^{*}$ is $\frac{1}{2} \square\left(\right.$ resp. $\left.\frac{1}{2} \Delta^{\mathscr{R}}\right)$.

Proof. The parallel translation $/ /_{0, t}^{E}$ satisfies the equation

$$
d^{\nabla^{h}} / /_{0, t}^{E}=h_{/ /_{0, t}^{E}}\left(d^{\nabla} X_{t}\right)
$$


which can be rewritten locally as

$$
d^{\nabla^{h}} / /_{0, t}^{E}=\sum_{i=1}^{m} e_{i}^{h}\left(/ /_{0, t}^{E}\right) d B_{t}^{i}
$$

where $\left(e_{i}\right)_{1<i<m}$ is a local section of the orthonormal frame bundle over $M$ and the processes $B^{i}$ are real-valued Brownian motions. The Stratonovich version of this equation is

$$
\delta / /_{0, t}^{E}=\sum_{i=1}^{m} e_{i}^{h}\left(/ /_{0, t}^{E}\right) \delta B_{t}^{i}-\frac{1}{2} \sum_{i=1}^{m} \nabla_{e_{i}^{h}}^{h} e_{i}^{h}\left(/ /_{0, t}^{E}\right) d t
$$

Hence $/ /_{0, t}^{E}$ is seen to be a diffusion with generator

$$
\frac{1}{2} \sum_{i=1}^{n}\left(\left(e_{i}^{h}\right)^{2}-\nabla_{e_{i}^{h}}^{h} e_{i}^{h}\right)=\frac{1}{2} \sum_{i=1}^{n}\left(\left(e_{i}^{h}\right)^{2}-\left(\nabla_{e_{i}} e_{i}\right)^{h}\right) .
$$

But if $\alpha$ is a section of $E^{*}, u$ a section of $T M$ with horizontal lift $u^{h}, s$ a section of $E$ with vertical lift $s^{v}$, and $r$ a section of $E$, then

$$
u^{h}(\alpha)(r)=\left\langle\nabla_{u}^{E^{*}} \alpha, r\right\rangle \quad \text { and } \quad s^{v}(\alpha)(r)=\langle\alpha, s\rangle .
$$

The second equality in Eq. (5.13) is obvious. Let us explain the first equality: if $t \mapsto r(t)$ is $C^{1}$ with $r(0)=r_{0} \in E_{x_{0}}$ and $\dot{r}(0)=u^{h}\left(r_{0}\right)$, then

$$
u^{h}(\alpha)\left(r_{0}\right)=\left.\frac{d}{d t}\right|_{t=0} \alpha(r(t))=\left\langle\nabla_{u_{x_{0}}}^{E^{*}} \alpha, r_{0}\right\rangle+\left\langle\alpha\left(x_{0}\right), \nabla_{u_{x_{0}}}^{E} r\right\rangle=\left\langle\nabla_{u_{x_{0}}}^{E^{*}} \alpha, r_{0}\right\rangle .
$$

The first equation of (5.13) then gives the result for the generator of $/ /_{0,}^{E} e$.

On the other hand, by Eqs. (5.5), (5.1) (5.2), and with the same calculation as before, $\Theta_{0, t} e$ has generator

$$
\frac{1}{2} \sum_{i=1}^{n}\left(\left(e_{i}^{h}\right)^{2}-\left(\nabla_{e_{i}} e_{i}\right)^{h}\right)-\frac{1}{2}(\operatorname{tr} \mathscr{R})^{v} .
$$

The second part of (5.13) with $s=\operatorname{tr} \mathscr{R}\left(r^{\prime}\right)$ gives

$$
\left(\operatorname{tr} \mathscr{R}\left(r^{\prime}\right)\right)^{v}(\alpha)(r)=\left\langle\alpha, \operatorname{tr} \mathscr{R}\left(r^{\prime}\right)\right\rangle=-\left\langle\operatorname{tr} \mathscr{R} \alpha, r^{\prime}\right\rangle .
$$

This combined with the same argument as before for the horizontal part gives the claim for the generator of $\Theta_{0, t} e$.

As a consequence, we have the following result.

Theorem 5.8. Let $M$ be a Riemannian manifold with the Levi-Civita connection $\nabla$ and $\pi: E \rightarrow M$ a vector bundle equipped with a covariant derivative $\nabla^{E}$. Let $a:[0, T] \times M \rightarrow E^{*}$ be a smooth solution to the heat equation $\frac{d}{d t} a=\frac{1}{2} \Delta^{\mathscr{R}} a$. Then

$$
a\left(T-t, X_{t}\right), \quad 0 \leq t \leq T,
$$

is a $\nabla^{\mathscr{S}}$-martingale in $E^{*}$ for any Brownian motion $X$ on $M$.

In particular, a differential form $a \in \Gamma\left(E^{*}\right)$ is harmonic (i.e. $\Delta^{\mathscr{R}} a=0$ ) if and only if a $(X)$ is $a \nabla^{\mathscr{S}}$-martingale for any Brownian motion $X$ in $M$. 
Proof. This is a direct consequence of Proposition 5.7 which gives the generator of $\Theta_{0, \text {. }}$. and Proposition 5.6 which characterizes $\nabla^{\mathscr{S}}$-transports in $E^{*}$, together with Proposition 5.3 which characterizes martingales.

Identifying $\left(E^{*}\right)^{*}$ with $E$ and using again Proposition 5.6 gives immediately the following result:

Corollary 5.9. Let $M$ be a Riemannian manifold with the Levi-Civita connection $\nabla$ and $\pi: E \rightarrow M$ a vector bundle equipped with a covariant derivative $\nabla^{E}$. Let $a:[0, T] \times M \rightarrow E$ be a smooth solution to the heat equation $\frac{d}{d t} a=\frac{1}{2} \Delta^{\mathscr{R}} a$. Then

$$
a\left(T-t, X_{t}\right), \quad 0 \leq t \leq T,
$$

is $a \nabla^{\mathscr{S}}$-martingale in $E$ for any Brownian motion $X$ on $M$.

\section{Complete Lifts to Cotangent Bundles}

Let $(M, \nabla)$ be a manifold endowed with a torsion-free connection $\nabla$. On $T^{*} M$ consider the induced dual covariant derivative, as in Remark 4.3. In this situation Proposition 4.1 gives rise to a horizontal connection $\nabla^{h *}$ on $T^{*} M$, i.e. a covariant derivative on $T T^{*} M$.

In [19] the authors introduce a connection on $T^{*} M$ which we denote here by $\nabla^{b}$ and which they call the complete lift of $\nabla$ : by definition it is the Levi-Civita connection for the pseudo-Riemannian metric $g^{b}$ on $T^{*} M$ given by

$$
d s^{2}=2 d x^{i}\left(d p_{i}-p_{k} \Gamma_{j i}^{k} d x^{j}\right)
$$

where $\left(x^{i}, p_{i}\right)$ are the local coordinates. This pseudo-Riemannian metric $g^{b}$ is alternatively described as

$$
g^{b}\left(X^{c}, Y^{c}\right)=-\left(\nabla_{X} Y+\nabla_{Y} X\right)^{v}, \quad X, Y \in \Gamma(T M) .
$$

Here $X^{c}, Y^{c} \in \Gamma\left(T T^{*} M\right)$ denote the complete lifts of $X, Y$, and $X^{v} \in C^{\infty}\left(T^{*} M\right)$, $X^{v}(\alpha):=\alpha\left(X_{\pi(\alpha)}\right)$, the vertical lift of $X$, see [19], chapt. VII for details.

Define the section $\mathscr{R} \in \Gamma\left(T^{*} M \otimes T^{*} M \otimes \operatorname{End}(T M)\right)$ by the formula

$$
\mathscr{R}(X, Y) J=R(J, X) Y, \quad X, Y, J \in T_{x} M .
$$

Recall that $\mathscr{R}$ is associated with the complete lift $\nabla^{c}$ of $\nabla$ in $T M$.

A calculation shows that for every $V, W \in \Gamma\left(T T^{*} M\right), p \in T^{*} M$,

$$
\nabla_{V_{p}}^{b} W=\nabla_{V_{p}}^{h *} W+v_{p}\left(\left\langle p, R\left(\cdot, \pi_{*} V_{p}\right) \pi_{*} W_{p}\right\rangle\right)
$$

(see for instance [19], chapt. VIII; in [19], the authors define $\nabla^{h *}$ by means of (6.2) and prove then the relations in Proposition 4.1). Hence $\nabla^{b}$ is a connection of the type $\nabla^{\mathscr{S}}$, as defined in Section 5, with

$$
\mathscr{S}\left(A_{\alpha}, B_{\alpha}\right)=v_{\alpha}\left(\left(\mathscr{R}\left(\pi_{*} A_{\alpha}, \pi_{*} B_{\alpha}\right)\right)^{*} \alpha\right), \quad A, B \in T_{\alpha} T^{*} M .
$$

$\nabla^{b}$ does not coincide with the adjoint of $\nabla^{c}$ as in Section 5. Indeed, the adjoint of $\nabla^{c}$ is $\nabla^{(-\mathscr{S})}$ with $\mathscr{S}$ as in (6.3). The adjoint of $\nabla^{c}$ will also be called the complete lift of $\nabla$ in $T^{*} M$, and denoted by $\nabla^{c}$ when there is no risk of confusion.

In the case where $(M, g)$ is a Riemannian manifold and $\nabla$ the Levi-Civita connection associated with the metric $g$, the complete lift $\nabla^{c}$ of $\nabla$ on $T M$ is the Levi-Civita 
connection associated with the complete lift $g^{c}$ on $T M$ of $g$ (see [19], $g^{c}$ is in fact a pseudo-metric). In this situation, it is easily proved that the map

$$
\text { b : }\left(T M, g^{c}\right) \rightarrow\left(T^{*} M, g^{b}\right), \quad u \mapsto g(u, \cdot)
$$

is an isometry.

Proposition 6.1. Let $\nabla$ be the Levi-Civita connection on a Riemannian manifold $M$. Then a $T M$-valued continuous semimartingale $J$ is a $\nabla^{c}$-martingale if and only if the $T^{*} M$-valued process $J^{\mathrm{b}}$ is a $\nabla^{\mathrm{b}}$-martingale.

Proof. This is a consequence of the fact that $b$ is an isometry. One can also give a proof based on symmetry relations satisfied by the curvature tensor. Let $J$ be a $T M$-valued semimartingale such that $X=\pi(J)$ is a $\nabla$-martingale. Then by Eqs. (4.5) and (4.6), $J$ is a $\nabla^{c}$-martingale if and only if for every $u \in T_{X_{0}} M$

$$
\left\langle/ /_{0, t}^{-1} J_{t}, u\right\rangle+\frac{1}{2} \int_{0}^{t}\left\langle/ /_{0, s}^{-1} R\left(J_{s}, d X_{s}\right) d X_{s}, u\right\rangle
$$

is a local martingale. But since $/ / 0, t$ is an isometry, (6.5) may also be written as

$$
\left(J_{t}^{\mathrm{b}} / /_{0, t}, u\right)+\frac{1}{2} \int_{0}^{t}\left\langle R\left(J_{s}, d X_{s}\right) d X_{s}, / /_{0, s} u\right\rangle,
$$

and using the relation $\langle R(A, B) C, D\rangle=\langle R(D, C) B, A\rangle$, this writes

$$
\left(J_{t}^{\mathrm{b}} / /_{0, t}, u\right)+\frac{1}{2} \int_{0}^{t}\left(J^{\mathrm{b}}, R\left(/ /_{0, s} u, d X_{s}\right) d X_{s}\right) .
$$

Hence (6.5) is a local martingale for all $u$ if and only if

$$
J_{t}^{\mathrm{b}} / /_{0, t}+\frac{1}{2} \int_{0}^{t}\left(J^{\mathrm{b}}, R\left(/ / /_{0, s}, d X_{s}\right) d X_{s}\right)
$$

is a local martingale, or if and only if $J^{b}$ is a $\nabla^{b}$ martingale. This achieves the proof.

\section{Complete Lifts to Exterior Bundles}

In this section $(M, \nabla)$ is a manifold endowed with a connection possibly with torsion $T$. We denote by $\hat{\nabla}$ the adjoint connection given by $\nabla-\hat{\nabla}=T$ and by $\hat{R}$ the associated curvature tensor. Let $E=\wedge^{p} T M$. On $E$ there is a covariant derivative inherited from $\nabla$, and hence, by Proposition 4.1, a covariant derivative $\nabla^{h}$ on $T E$. Let us define a section $\mathscr{R}^{E} \in \Gamma\left(T^{*} M \otimes T^{*} M \otimes\right.$ End $\left.E\right)$ as the linear extension of

$$
\begin{aligned}
\mathscr{R}^{E}(u, w) v_{1} \wedge \ldots \wedge v_{p} \\
=\sum_{k=1}^{p} v_{1} \wedge \ldots \wedge\left(R\left(v_{k}, u\right) w+\nabla_{u} T\left(v_{k}, w\right)\right) \wedge \ldots \wedge v_{p} \\
\quad-\sum_{1 \leq \ell<k \leq p}(-1)^{k+\ell} \hat{R}\left(v_{k}, v_{\ell}\right) u \wedge w \wedge v_{1} \wedge \ldots \wedge \widehat{v}_{\ell} \wedge \ldots \wedge \widehat{v}_{k} \wedge \ldots \wedge v_{p}
\end{aligned}
$$

for $u, w, v_{1}, \ldots, v_{p} \in T_{x} M$. Note that in case $p=1$ this formula coincides with the $\mathscr{R}$ in (5.3). Also note that one has to verify that this definition makes sense, i.e. that the right hand side vanishes when $v_{i}=v_{j}, i \neq j$. 
Definition 7.1. The complete lift of $\nabla$ to $E$, denoted by $\nabla^{c}$, is the connection $\nabla^{\mathscr{S}}$ on $E$ associated with $\mathscr{R}=\mathscr{R}^{E}$, as defined above, and $\mathscr{T}=0$.

The complete lift of $\nabla$ to $E^{*}$, again denoted by $\nabla^{c}$, is the connection $\nabla^{\mathscr{S}}$ on $E^{*}$ associated with $\mathscr{R}^{E^{*}}=-\left(\mathscr{R}^{E}\right)^{*}$, also denoted by $\mathscr{R}$, and with $\mathscr{T}=0$.

If $\nabla$ is torsion-free, then the above definition extends the definitions for $p=1$ given in Sections 2 and 6.

Lemma 7.2. Assume $\nabla$ is torsion-free. If $u, w, v_{1}, \ldots, v_{p} \in T_{x} M$ and $\alpha \in E_{x}^{*}$, then $\mathscr{R}(u, w) \alpha \in E_{x}^{*}$ satisfies

$$
\mathscr{R}(u, w) \alpha\left(v_{1}, \ldots, v_{p}\right)=\sum_{k=1}^{p} R\left(v_{k}, u\right) \alpha\left(v_{1}, \ldots, v_{k-1}, w, v_{k+1}, \ldots, v_{p}\right) .
$$

Note that (7.1) gives in the particular case $\alpha=\alpha_{1} \wedge \ldots \wedge \alpha_{p}$

$$
\begin{array}{r}
\mathscr{R}(u, w) \alpha\left(v_{1}, \ldots, v_{p}\right)=-\sum_{k, \ell=1}^{p} \alpha_{1} \wedge \ldots \wedge \alpha_{\ell-1} \wedge\left\langle\alpha_{\ell}, R\left(v_{k}, u\right) \cdot\right\rangle \wedge \alpha_{\ell+1} \wedge \ldots \\
\ldots \wedge \alpha_{p}\left(v_{1}, \ldots, v_{k-1}, w, v_{k+1}, \ldots, v_{p}\right) .
\end{array}
$$

Proof. Since by definition

$$
\left\langle\mathscr{R}(u, w) \alpha,\left(v_{1}, \ldots, v_{p}\right)\right\rangle=-\left\langle\alpha, \mathscr{R}(u, w)\left(v_{1} \wedge \ldots \wedge v_{p}\right)\right\rangle,
$$

it is sufficient to prove that

$$
\mathscr{R}(u, w) v_{1} \wedge \ldots \wedge v_{p}=\sum_{k=1}^{p} R\left(v_{k}, u\right)\left(v_{1} \wedge \ldots \wedge w \wedge \ldots \wedge v_{p}\right) .
$$

But by developing the right hand side of (7.2), isolating the terms with $R\left(v_{k}, u\right) w$ and putting together terms with the same pair of indices, we obtain

$$
\begin{array}{r}
\sum_{k=1}^{p} R\left(v_{k}, u\right)\left(v_{1} \wedge \ldots \wedge w \wedge \ldots \wedge v_{p}\right)=\sum_{k=1}^{p} v_{1} \wedge \ldots \wedge R\left(v_{k}, u\right) w \wedge \ldots \wedge v_{p} \\
-\sum_{1 \leq \ell<k \leq p}(-1)^{\ell+k}\left(R\left(v_{k}, u\right) v_{\ell}-R\left(v_{\ell}, u\right) v_{k}\right) \wedge w \wedge v_{1} \wedge \ldots \wedge \widehat{v}_{\ell} \wedge \ldots \\
\ldots \wedge \widehat{v}_{k} \wedge \ldots \wedge v_{p} .
\end{array}
$$

The result follows by using the Bianchi identity

$$
R\left(v_{k}, u\right) v_{\ell}-R\left(v_{\ell}, u\right) v_{k}=R\left(v_{k}, v_{\ell}\right) u \text {. }
$$

As a consequence of Lemma 7.2 and [9] Proposition 8.7, we can state:

Corollary 7.3. Assume that $M$ is a Riemannian manifold equipped with its Levi-Civita connection. Let $E=\wedge T M$ and $\mathscr{R}$ be as in (7.1). Let $\Delta:=-\left(d+d^{*}\right)^{2}$ be the Laplacian on $\Gamma\left(E^{*}\right)$, and let $\Delta^{\mathscr{R}}=\square+\operatorname{tr} \mathscr{R}$ be the $\mathscr{R}$-Laplacian on $\Gamma\left(E^{*}\right)$ defined in Section 5 . Then

$$
\Delta=\Delta^{\mathscr{R}}
$$


Remark 7.4. Under the assumptions of Corollary 7.3 the following formula holds:

$$
\begin{aligned}
& \mathscr{R}(u, w) v_{1} \\
& \wedge \ldots \wedge v_{p} \\
&=\sum_{k=1}^{p} v_{1} \wedge \ldots \wedge R\left(v_{k}, u\right) w \wedge \ldots \wedge v_{p} \\
&-\sum_{1 \leq \ell<k \leq p}(-1)^{k+\ell} R\left(v_{k}, v_{\ell}\right) u \wedge w \wedge v_{1} \wedge \ldots \wedge \widehat{v}_{\ell} \wedge \ldots \wedge \widehat{v}_{k} \wedge \ldots \wedge v_{p}
\end{aligned}
$$

The second term on the right says that $\mathscr{R}$ is not a tensor product as defined in formula (5.10). In other words, the tensor product of deformed parallel transports in $T M$ is not the deformed parallel transport associated with $\Delta$. This has been pointed out in [16] p. 196.

Let $E=\wedge^{p} T M$. For every $x \in M$, let $X(x)$ be an $M$-valued semimartingale with starting point $x$. Assume that $X$ is differentiable with respect to $x$ and denote by $T X$ the derivative of $X$. We want to calculate the drift of the process $T X^{\wedge p}$ with respect to the connection $\nabla^{c}$, and to find sufficient conditions under which the fact that $X$ is a $\nabla$-martingale implies that $T X^{\wedge p}$ is a $\nabla^{c}$-martingale. We have

$$
\begin{aligned}
D\left(T X^{\wedge p}\right):= & / /_{0, .}^{E} d\left(\left(/ /\left.\right|_{0} ^{E} .\right)^{-1} T X^{\wedge p}\right)=/ /_{0, .}^{E} d\left(/ /_{0, .}^{-1} T X\right)^{\wedge p} \\
= & \sum_{k=1}^{p} T X_{1} \wedge \ldots \wedge D T X_{k} \wedge \ldots \wedge T X_{p} \\
& \quad+\sum_{1 \leq \ell<k \leq p} T X_{1} \wedge \ldots \wedge D T X_{\ell} \wedge \ldots \wedge D T X_{k} \wedge \ldots \wedge T X_{p} .
\end{aligned}
$$

Consider the particular case when $X$ solves an equation of the type

$$
d^{\nabla} X=A(X) d B+b(X) d t
$$

where $B$ is an $\mathbb{R}^{r}$-valued Brownian motion, $A \in \Gamma\left(\mathbb{R}^{r} \otimes T M\right), b \in \Gamma(T M)$. Differentiating with the help of Theorem 2.2 yields

$$
d^{\nabla^{c}} T X=A^{c}(T X) d B+b^{c}(T X) d t
$$

or with Eq. (5.3)

$$
\begin{aligned}
d^{\nabla^{h}} T X= & A^{c}(T X) d B+b^{c}(T X) d t \\
& -\frac{1}{2} v_{T X}(R(T X, d X) d X+\nabla T(d X, T X, d X)+T(D T X, d X)) d t .
\end{aligned}
$$

Projecting onto the vertical part gives

$$
\begin{aligned}
D T X= & \hat{\nabla}_{T X} A d B+\hat{\nabla}_{T X} b d t \\
& -\frac{1}{2}(R(T X, d X) d X+\nabla T(d X, T X, d X)+T(D T X, d X)) d t .
\end{aligned}
$$

Definition 7.5. We say that $(A, \nabla)$ satisfies the condition (LJW) if $\operatorname{Im} A$ is a subbundle of $T M$ and the restriction of the adjoint connection $\hat{\nabla}$ to sections of $\operatorname{Im} A$ is the Le JanWatanabe covariant derivative induced by $A$. 
Recall that the Le Jan-Watanabe covariant derivative $\nabla^{\mathrm{LJW}}$ on $\operatorname{Im} A$ is characterized as follows (see [10]): if $Z \in \Gamma(\operatorname{Im} A), v_{0} \in T_{x_{0}} M$, then

$$
\nabla_{v_{0}}^{\mathrm{LJW}} Z=A\left(x_{0}\right) d\left(A^{*}(\cdot) Z(\cdot)\right)\left(v_{0}\right)
$$

where the adjoint $A^{*}$ is defined with respect to an induced metric on $\operatorname{Im} A$ which makes each $A^{*}(x)$ an isometric embedding into $\mathbb{R}^{r}$. The Le Jan-Watanabe covariant derivative satisfies the property: if $x \in M, e \in(\operatorname{ker} A(x))^{\perp}, w \in T_{x} M$, then $\nabla_{w}^{\mathrm{LJW}} A(\cdot) e=0$ ([10] Proposition 1.1.1).

Note that our notations are not the same as in [10]. Our $\nabla$ is the adjoint of an extension, as in their Proposition 1.3.1, of their $\breve{\nabla}$. Hence our restriction of $\hat{\nabla}$ to $\operatorname{Im} A$ is their $\breve{\nabla}$.

The most important example is given by gradient Brownian systems: $M$ is a Riemannian manifold isometrically immersed in $\mathbb{R}^{r}, A(x)$ the orthogonal projection of $\mathbb{R}^{r}$ to $T_{x} M$ and $\nabla^{\mathrm{LJW}}$ the Levi-Civita connection ([10] Example 1B).

Note that given $A$ such that $\operatorname{Im} A$ is a subbundle of $T M$, the existence of $\nabla$ such that $(A, \nabla)$ satisfies (LJW) is guaranteed by [10] Proposition 1.3.1.

Assume that $(A, \nabla)$ satisfies (LJW). Let $x \in M$. Choosing an orthonormal basis of $\mathbb{R}^{r}$ which splits into an orthonormal basis of ker $A(x)$ and an orthonormal basis of $(\operatorname{ker} A(x))^{\perp}$, we see that $\operatorname{tr} \hat{\nabla} A(x) \otimes A(x)=0$. But with the expression of $D T X$ in Eq. (7.4) we get $D T X \otimes d X=\operatorname{tr} \hat{\nabla} A(X) \otimes A(X) d t$; we conclude that

$$
D T X \otimes d X \equiv 0 .
$$

As a consequence, using the relation

$$
R(v, u) u+\left(\nabla_{u} T\right)(v, u)=\hat{R}(v, u) u, \quad u, v \in T_{x} M,
$$

and again Eq. (7.4), we get

$$
D T X=\hat{\nabla}_{T X} A(X) d B+\hat{\nabla}_{T X} b(X) d t-\frac{1}{2} \sum_{i=1}^{m} \hat{R}\left(T X, A_{i}(X)\right) A_{i}(X) d t,
$$

and this gives

$$
\begin{aligned}
& D\left(T X^{\wedge p}\right)= \sum_{k=1}^{p} T X_{1} \wedge \ldots \wedge \hat{\nabla}_{T X_{k}} A(X) d B \wedge \ldots \wedge T X_{p} \\
&+\sum_{k=1}^{p} T X_{1} \wedge \ldots \wedge \hat{\nabla}_{T X_{k}} b(X) d t \wedge \ldots \wedge T X_{p} \\
&+\sum_{k=1}^{p} T X_{1} \wedge \ldots \wedge\left(-\frac{1}{2} \sum_{i=1}^{m} \hat{R}\left(T X_{k}, A_{i}(X)\right) A_{i}(X) d t\right) \wedge \ldots \wedge T X_{p} \\
&+\sum_{1 \leq \ell<k \leq p}(-1)^{k+\ell} \sum_{i=1}^{m} \hat{\nabla}_{T X_{k}} A_{i}(X) \wedge \hat{\nabla}_{T X_{\ell}} A_{i}(X) \wedge T X_{1} \wedge \ldots \\
& \ldots \wedge \widehat{T X}_{\ell} \wedge \ldots \wedge \widehat{T X}_{k} \wedge \ldots \wedge T X_{p} d t .
\end{aligned}
$$

The displayed equation in the next proposition already appears in the proof of Theorem 3.3 .8 in [10]. 
Proposition 7.6. Assume that $(A, \nabla)$ satisfies (LJW). Then

$$
\begin{aligned}
D\left(T X^{\wedge p}\right)= & \sum_{k=1}^{p} T X_{1} \wedge \ldots \wedge \hat{\nabla}_{T X_{k}} A(X) d B \wedge \ldots \wedge T X_{p} \\
& +\sum_{k=1}^{p} T X_{1} \wedge \ldots \wedge \hat{\nabla}_{T X_{k}} b(X) d t \wedge \ldots \wedge T X_{p} \\
& -\frac{1}{2} \sum_{i=1}^{m} \mathscr{R}\left(A_{i}(X), A_{i}(X)\right) T X^{\wedge p} d t .
\end{aligned}
$$

In particular, if $X$ is a $\nabla$-martingale, then $T X^{\wedge p}$ is a $\nabla^{c}$-martingale.

Proof. For the claimed equation, considering the formula just before Proposition 7.6 and the definition of $\mathscr{R}^{E}$, it is sufficient to prove that for all $x \in M, v_{1}, v_{2} \in T_{x} M$

$$
\sum_{j=1}^{m} \hat{R}\left(v_{1}, v_{2}\right) A_{j} \wedge A_{j}=2 \sum_{j=1}^{m} \hat{\nabla}_{v_{1}} A_{j} \wedge \hat{\nabla}_{v_{2}} A_{j},
$$

which is a consequence of [10], Corollary C.5. If $X$ is a $\nabla$-martingale, then $b \equiv 0$, and we have by (5.4) and (4.8)

$$
\begin{aligned}
d^{\nabla^{c}} T X^{\wedge p} & =d^{\nabla^{h}} T X^{\wedge p}+v_{T X^{\wedge p}}\left(\frac{1}{2} \mathscr{R}(d X, d X) T X^{\wedge p}\right) \\
& =h_{T X^{\wedge p}}\left(d^{\nabla} X\right)+v_{T X^{\wedge p}}\left(D\left(T X^{\wedge p}\right)\right)+v_{T X^{\wedge p}}\left(\frac{1}{2} \mathscr{R}(d X, d X) T X^{\wedge p}\right) \\
& =h_{T X^{\wedge p}}\left(d^{\nabla} X\right)+\sum_{k=1}^{p} T X_{1} \wedge \ldots \wedge \hat{\nabla}_{T X_{k}} A(X) d B \wedge \ldots \wedge T X_{p}
\end{aligned}
$$

which shows that $T X^{\wedge p}$ is a $\nabla^{c}$-martingale.

\section{Complete Lifts to Dirac Bundles}

Let $D$ be the Dirac operator on the spinor bundle $F$ over a spin manifold $M$ and $-D^{2}=\square+\mathcal{R}$ the Weitzenböck decomposition of its square. We want to define a natural connection $\nabla^{c}$ on $F$, such that a section ("spinor field") $a \in \Gamma(F)$ is harmonic (i.e., $a \in \operatorname{ker} D^{2}$ ) if and only if $a(X)$ is a $\nabla^{c}$-martingale for any Brownian motion $X$ in $M$.

More generally, we shall deal with the following context.

Definition 8.1. Let $M$ be a Riemannian manifold with Levi-Civita connection and $\pi: E \rightarrow M$ a Riemannian vector bundle over $M$, endowed with a Riemannian connection $\nabla^{E}$ :

$$
X\langle a, b\rangle=\left\langle\nabla_{X}^{E} a, b\right\rangle+\left\langle a, \nabla_{X}^{E} b\right\rangle, \quad a, b \in \Gamma(E), X \in \Gamma(T M) .
$$

Then $E$ is said to be a Dirac bundle if it carries a "Clifford action"

$$
c \in \Gamma(\operatorname{Hom}(T M \otimes E, E)),
$$

written as $v \cdot a:=c(v \otimes a) \in \Gamma(E)$ for $v \in \Gamma(T M)$ and $a \in \Gamma(E)$, such that 
(i) $\langle v \cdot a, b\rangle=-\langle a, v \cdot b\rangle$,

(ii) $\langle v \cdot a, v \cdot b\rangle=\|v\|^{2}\langle a, b\rangle$,

(iii) $\nabla^{E}(v \cdot a)=\left(\nabla^{T M} v\right) \cdot a+v \cdot\left(\nabla^{E} a\right)$

for all $v \in \Gamma(T M)$ and $a, b \in \Gamma(E)$. The operator

$$
D: \Gamma(E) \stackrel{\nabla^{E}}{\longrightarrow} \Gamma\left(T^{*} M \otimes E\right) \stackrel{\sim}{\longrightarrow} \Gamma(T M \otimes E) \stackrel{c}{\longrightarrow} \Gamma(E)
$$

is called Dirac operator of $E$ and $\Delta=-D^{2}$ its Laplacian.

Definition 8.1 is equivalent to the one in [13], p. 114. Note that if, for $v \in T_{x} M$, the linear map $c(v \otimes \cdot): E_{x} \rightarrow E_{x}$ is denoted by $c(v)$, then conditions (i) and (ii) amount to saying $c(v)^{*}=-c(v)$ and $c(v) c(w)+c(w) c(v)=-2\langle v, w\rangle$ id.

For such generalized Laplacians $\Delta$ there is a Lichnerowicz-Weitzenböck decomposition

$$
\Delta a=\square a+\mathcal{R} a
$$

where in terms of the curvature tensor $R_{x}: T_{x} M \times T_{x} M \rightarrow \operatorname{End}\left(E_{x}\right)$ :

$$
\mathcal{R}_{x} a=-\frac{1}{2} \sum_{i, j} e_{i} \cdot e_{j} \cdot R_{x}\left(e_{i}, e_{j}\right) a
$$

for any orthonormal basis $\left(e_{1}, \ldots, e_{n}\right)$ of $T_{x} M$ (see [13], p. 155).

Example 8.2. (a) (the de Rham operator) The exterior bundle $E=\wedge T^{*} M$ of a Riemannian manifold with Clifford action $v \cdot a=v^{b} \wedge a-v^{b}\llcorner a$ and Levi-Civita connection is a Dirac bundle, where by definition $v^{b}=\langle v, \cdot\rangle$. The associated Dirac operator is $D=d+d^{*}$ and $\Delta=-D^{2}=-\left(d d^{*}+d^{*} d\right)$ is the de Rham-Hodge Laplacian on $E$.

(b) (the Dirac operator on a spin manifold) If $M$ is an even-dimensional spin manifold, its spinor bundle $F$ with the Levi-Civita connection is a Dirac bundle. The associated Dirac operator $D$ is referred to as the Dirac operator on $M$.

(c) (the Dirac operator of a twisted spinor bundle) The spinor bundle $F$ is tensored with an auxiliary Riemannian/hermitian vector bundle $\xi$ over $M$ to give a Dirac operator of the form

$$
D: \Gamma(F \otimes \xi) \stackrel{\nabla^{F \otimes \xi}}{\longrightarrow} \Gamma\left(T^{*} M \otimes F \otimes \xi\right) \stackrel{\sim}{\longrightarrow} \Gamma(T M \otimes F \otimes \xi) \stackrel{c \otimes 1}{\longrightarrow} \Gamma(F \otimes \xi) .
$$

Again the identification in the middle is given by the metric.

(d) (the $\bar{\partial}$-operator on a Kähler manifold) e.g. [7], p. 135.

Given the situation of Definition 8.1, we now investigate connections $\nabla^{c}$ on $T E$ such that $a(X)$ is a $\nabla^{c}$-martingale for $a \in \Gamma(E)$ harmonic and $X$ a Brownian motion on $M$. Such connections will be possible candidates for the complete lift $\nabla^{c}$ of $\nabla$.

First of all note that one may modify Definition 7.1 by means of the Weitzenböck term (8.4) as follows: For $u, v \in T_{x} M$ and $a \in E_{x}$ let

$$
\mathscr{R}(u, v) a=-\sum_{j} v \cdot e_{j} \cdot R_{x}\left(u, e_{j}\right) a \in E_{x}
$$

and define for $Y \in \Gamma(T E), X \in T_{a} E, a \in E$,

$$
\nabla_{X}^{c} Y=\nabla_{X}^{h} Y+v_{a}\left(\mathscr{R}\left(\pi_{*} X, \pi_{*} Y\right) a\right) .
$$


This obviously gives a connection $\nabla^{c}$ with the wanted properties which however does not coincide with the connections on exterior bundles as defined in Section 7, when the latter are considered as special instances of Clifford bundles. In addition, there is a plenty of other connections satisfying the same conditions.

The particular case of exterior bundles already suggests that in case the Dirac bundle $E$ inherits a natural graduation or filtration, the connection should respect this additional structure.

Coming back to the case of the exterior bundle $E=\wedge T^{*} M$ of Example (8.2) (a), let $c(v)=C_{v}-A_{v}$ where $C_{v} a=v^{b} \wedge a, A_{v} a=v^{b}\left\llcorner a\right.$ for $v \in T_{x} M, a \in E_{x}$, and let $\operatorname{pr}^{p}: E \rightarrow E$ denote the projection onto $E^{p}:=\wedge^{p} T^{*} M$. Setting

$$
\begin{aligned}
& c^{+}(v)\left|E^{p}=\operatorname{pr}^{p+1} c(v)\right| E^{p} \\
& c^{-}(v)\left|E^{p}=\operatorname{pr}^{p-1} c(v)\right| E^{p},
\end{aligned}
$$

we get $c^{+}(v)=C_{v}$ and $c^{-}(v)=-A_{v}$. Moreover, it is straightforward to check that by defining

$$
\mathscr{R}^{\prime}(u, v) a=-\sum_{i} c^{-}(v) c^{+}\left(e_{i}\right) R\left(u, e_{i}\right) a \in E_{x}
$$

we get

$$
\mathscr{R}^{\prime}(u, v)=\mathscr{R}(u, v)+R(u, v)
$$

where $\mathscr{R}$ is defined by Eq. (7.1). Taking into account the antisymmetry of the curvature tensor $R(u, v)$, we see that Eqs. (8.6) and (8.7) define a connection $\nabla^{c}$ on the exterior bundle $E=\wedge T^{*} M$ which coincides up to torsion with the one defined in Section 7. In particular, both connections yield the same class of martingales.

\section{Martingales in the Tangent Space Related to Harmonic Maps}

Let $L=\frac{1}{2} \sum_{i=1}^{r} A_{i}^{2}+A_{0}$ be a differential operator on a manifold $M$ with a connection. Assume that for every $x \in M$, one can choose $r$ and the vector fields $A_{0}, \ldots, A_{r}$ in such a way that for all $i \geq 1$, either $A_{i}(x)=0$ or $\hat{\nabla} A_{i}(x)=0$ (this is a local property). Note this is possible for instance when $M$ is a Riemannian manifold, $\nabla$ is the Levi-Civita connection and $L=\frac{1}{2} \Delta+b$, or more generally, when $(A, \nabla)$ satisfies (LJW) (just make a rotation, depending on $x$, of the orthonormal basis in $\mathbb{R}^{r}$ ).

Let $N$ be another manifold with a connection also denoted by $\nabla$. For $T>0$ let $u: M \times[0, T] \rightarrow N$ be a smooth solution to the heat equation, i.e. $u(t-\cdot, X$.$) is$ an $N$-valued $\nabla$-martingale for every diffusion $X$ on $M$ with generator $L$ and every $t \in] 0, T]$.

Let $X(x)$ be a diffusion with generator $L$, satisfying $X_{0}(x)=x$ and

$$
\delta X(x)=A(X(x)) \delta B+A_{0}(X(x)) d t
$$

or in the Itô form

$$
d^{\nabla} X(x)=A(X(x)) d B+b(X(x)) d t
$$

with $b=A_{0}+\frac{1}{2} \sum_{i=1}^{r} \nabla_{A_{i}} A_{i}$. Here $B$ is a Brownian motion taking values in $\mathbb{R}^{r}$. 
Differentiating Eq. (9.1) along with Theorem 2.2 gives

$$
d^{\nabla^{c}} T X(v)=A^{c}(T X(v)) d B+b^{c}(T X(v)) d t
$$

for every $v \in T M$, where $T X$ is a diffusion with generator

$$
L^{c}:=\frac{1}{2} \sum_{i=1}^{r}\left(A_{i}^{c}\right)^{2}+A_{0}^{c}
$$

(Note that $L^{c}$ depends on the particular choice for $A$, not just on the operator $L$.)

Let $T u$ denote the derivative of $u$ with respect to the second, and $\partial u$ the derivative with respect to the first variable. We know by Corollary 2.3 that $s \mapsto T u\left(t-s, X_{s}\right)\left(T X_{s}\right)$ is a $\nabla^{c}$-martingale in $T N$.

Let $\Theta^{M}$ be a $L\left(T_{x} M, T M\right)$-valued diffusion satisfying $\Theta_{0}^{M}=\mathrm{id}_{T_{x} M}$ and

$$
d^{\nabla^{c}} \Theta^{M}=A^{h}\left(\Theta^{M}\right) d B+b^{c}\left(\Theta^{M}\right) d t
$$

By means of Eq. (4.7) and relation $\hat{R}\left(\Theta^{M}, A_{i}\right) A_{i}=R\left(\Theta^{M}, A_{i}\right) A_{i}+\nabla T\left(A_{i}, \Theta^{M}, A_{i}\right)$, along with the fact that $D \Theta^{M}$ has finite variation, Eq. (9.3) can be rewritten as

$$
d^{\nabla^{h}} \Theta^{M}=A^{h}\left(\Theta^{M}\right) d B+b^{c}\left(\Theta^{M}\right) d t-\frac{1}{2} v_{\Theta^{M}}\left(\sum_{i=1}^{r} \hat{R}\left(\Theta^{M}, A_{i}\right) A_{i}\right)
$$

But since

$$
b^{c}=b^{h}+\gamma(\hat{\nabla} . b)=A_{0}^{h}+\frac{1}{2} \sum_{i=1}^{r} \nabla_{A_{i}^{h}}^{h} A_{i}^{h}+\gamma(\hat{\nabla} . b)
$$

where $\gamma\left(\hat{\nabla}_{.} b\right)(w)=v_{w}\left(\hat{\nabla}_{w} b\right)$ for $w \in T M$, we conclude that $\Theta^{M}$ is a diffusion with generator

$$
L^{\nabla}:=\frac{1}{2} \sum_{i=1}^{r}\left(A_{i}^{h}\right)^{2}+A_{0}^{h}-\frac{1}{2} \sum_{i=1}^{r} \gamma\left(\hat{R}\left(\cdot, A_{i}\right) A_{i}\right)+\gamma(\hat{\nabla} . b) .
$$

Since Eq. (9.3) and $b^{c}=b^{h}+\gamma(\hat{\nabla} . b)$ imply

$$
d^{\nabla^{c}} \Theta^{M}(v)=h_{\Theta^{M}(v)}\left(d^{\nabla} X\right)+v_{\Theta^{M}(v)}\left(\hat{\nabla}_{\Theta^{M}(v)} b\right) d t
$$

we see that $L^{\nabla}$ depends on $L$ and $\nabla$, but not on the particular choice for $A$. (Recall that $b$ is the first order part of $L$ with respect to the connection $\nabla$.)

Proposition 9.1. The process $T u(t-\cdot, X)\left(\Theta^{M}\right)$ is a $\nabla^{c}$-martingale in $T N$. 
Proof. In the following calculation we will use the identity $A^{h}=A^{c}-\gamma(\hat{\nabla} A)$. The geometric Itô equation for $T u(t-\cdot, X)\left(\Theta^{M}\right)$ writes

$$
\begin{array}{rl}
d^{\nabla^{c}} & T u(t-\cdot, X)\left(\Theta^{M}\right) \\
= & \left\langle T T u(t-\cdot, X), d^{\nabla^{c}} \Theta^{M}\right\rangle+\frac{1}{2} \nabla^{c} T T u(t-\cdot, X)\left(d^{\nabla^{c}} \Theta^{M} \otimes d^{\nabla^{c} \Theta^{M}}\right) \\
& -\partial T u(t-\cdot, X) d s \\
= & \left\langle T T u(t-\cdot, X) A^{h}\left(\Theta^{M}\right) d B+b^{c}\left(\Theta^{M}\right) d s\right\rangle-\partial T u(t-\cdot, X) d s \\
& +\frac{1}{2} \nabla^{c} T T u(t-\cdot, X)\left(A^{h}\left(\Theta^{M}\right) d B \otimes A^{h}\left(\Theta^{M}\right) d B\right) \\
\underline{m} & \left\langle T T u(t-\cdot, X), b^{c}\left(\Theta^{M}\right) d s\right\rangle \\
& +\frac{1}{2} \nabla^{c} T T u(t-\cdot, X)\left(A^{c}\left(\Theta^{M}\right) d B \otimes A^{c}\left(\Theta^{M}\right) d B\right) \\
& -\partial T u(t-\cdot, X) d s \\
& -\nabla^{c} T T u(t-\cdot, X)\left(A^{h}\left(\Theta^{M}\right) d B \otimes v_{\Theta^{M}}\left(\hat{\nabla}_{\Theta^{M}} A\right) d B\right) \\
& +\frac{1}{2} \nabla^{c} T T u(t-\cdot, X)\left(v_{\Theta^{M}}\left(\hat{\nabla}_{\Theta^{M}} A\right) d B \otimes v_{\Theta^{M}}\left(\hat{\nabla}_{\Theta^{M}} A\right) d B\right)
\end{array}
$$

where $\stackrel{m}{=}$ means again equality modulo differentials of local martingales. The sum of the first three lines of the r.h.s. vanishes by means of Eq. (9.2) since $T u(t-., X)(T X)$ is a $\nabla^{c}$-martingale for every $X$ as above, and therefore the geometric Itô formula for TuTX yields: for all $w \in T_{x} M$,

$0=\left\langle T T u(t-\cdot, x), b^{c}(w)\right\rangle+\frac{1}{2} \operatorname{tr} \nabla^{c} T T u(t-\cdot, x)\left(A^{c}(w) \otimes A^{c}(w)\right)-\partial T u(t-\cdot, x)$.

The last line of the r.h.s. vanishes since $T u$ is linear on the fibers, $\nabla^{c}$ is flat on the fibers, and hence $\nabla^{c} T T u\left(w_{1}, w_{2}\right)=0$ if $w_{1}$ and $w_{2}$ are vertical. To prove that the fourth line of the r.h.s. vanishes, we first remark that (since $\Theta^{M}$ is a diffusion)

$$
d^{\nabla^{c}} T u(t-\cdot, X)\left(\Theta^{M}\right) \stackrel{\mathrm{m}}{=} F\left(\cdot, \Theta^{M}\right) d t
$$

for some continuous map $F$ which does not depend on the choice of $A$. But the calculation above gives

$$
\begin{aligned}
& d^{\nabla^{c}} T u(t-\cdot, X)\left(\Theta^{M}\right) \\
& \quad \stackrel{\mathrm{m}}{=}-\nabla^{c} T T u(t-\cdot, X)\left(\sum_{i=1}^{r}\left(A_{i}^{h}\left(\Theta^{M}\right) \otimes v_{\Theta^{M}}\left(\hat{\nabla}_{\Theta^{M}} A_{i}\right)\right)\right) d t
\end{aligned}
$$

where the right hand side is continuous in $\Theta^{M}$. Consequently,

$$
F(\cdot, w)=-\nabla^{c} T T u(t-\cdot, x)\left(\sum_{i=1}^{r}\left(A_{i}^{h}(x) \otimes v_{w}\left(\hat{\nabla}_{w} A_{i}(x)\right)\right)\right)
$$

for all $x \in M$ and $w \in T_{x} M$. Now choosing $A$ such that for all $i \geq 1$, either $A_{i}(x)=0$ or $\hat{\nabla} A_{i}(x)=0$, we see that $F(\cdot, w)=0$, and as a conclusion, that $T u(t-\cdot, X) \Theta^{M}$ is a $\nabla^{c}$-martingale. 
Proposition 9.1 provides an alternative to the method of filtering out redundant noise, as discussed in [10]. Recall that the condition on the connection described at the beginning of the section is satisfied if $L=\frac{1}{2} \sum_{i=1}^{r} A_{i}^{2}+A_{0}$ and $(A, \nabla)$ satisfies condition (LJW), up to a rotation of the orthonormal basis in $\mathbb{R}^{r}$. This gives the following result:

Corollary 9.2. Let $(A, \nabla)$ satisfy (LJW) and

$$
L=\frac{1}{2} \sum_{i=1}^{r} A_{i}^{2}+A_{0}
$$

with $A_{0}=-\frac{1}{2} \sum_{i=1}^{r} \nabla_{A_{i}} A_{i}$. Let $u:(M, L) \rightarrow(N, \nabla)$ be a smooth harmonic map. Assume that the connections $\nabla$ in $M$ and $N$ are torsion-free.

Then Tu: $(M, L) \rightarrow\left(T^{*} M \otimes T N, \nabla^{c}\right)$ is harmonic, where $\nabla^{c}$ is the tensor product of the complete lifts of $\nabla$ in $T N$ and $T^{*} M$.

Remark 9.3. Replacing for instance $M$ by $M \times \mathbb{R}$ with the product connection $\tilde{\nabla}$ allows us to differentiate with respect to a parameter. We just have to verify that $(A, \tilde{\nabla})$ satisfies (LJW). We conclude that Corollary 9.2 is a generalization to harmonic maps of the fact that Jacobi fields are $\nabla^{c}$-geodesics.

Proof. Since $A_{0}=-\frac{1}{2} \sum_{i=1}^{r} \nabla_{A_{i}} A_{i}$ (which implies that any diffusion with generator $L$ is a $\nabla$-martingale), the term $b$ in (9.1) vanishes. As a consequence, the $\Theta^{M}$ satisfying Eq. (9.3) is the canonical $\nabla^{c}$-martingale along $X$. By Propositions 5.3 and 5.6 it is sufficient to prove that $\Theta^{N^{-1}} T u(X) \Theta^{M}$ is a local martingale, where $\Theta^{N}$ is the horizontal $\nabla^{c}$-martingale along $u(X)$. But since $T u(X) \Theta^{M}$ is a $\nabla^{c}$-martingale by Proposition 9.1, applying again Proposition 5.3 gives the result.

\section{Martingales in the Exterior Tangent Bundle Related to HARMONIC FORMS}

Let $(M, \nabla)$ be a manifold endowed with a connection possibly with torsion. Let $E=\wedge^{p} T M$ and let $\nabla^{c}$ be the complete lift of $\nabla$ to $E$, as defined in Section 7 . Let $L$ be the generator of an $M$-valued diffusion process $X$ solving

$$
d^{\nabla} X=A(X) d B
$$

where $A \in \Gamma\left(\mathbb{R}^{r} \otimes T M\right)$ and $B$ is an $\mathbb{R}^{r}$-valued Brownian motion. In particular, $X$ is assumed to be a $\nabla$-martingale. We are interested in the problem inverse to Proposition 9.1 of Section 9. Given a harmonic $p$-form $\alpha$ on $M$, we want to find conditions under which $\left\langle\alpha, T X^{\wedge p}\right\rangle$ is also a local martingale. The following result is a consequence of [10], Theorem 2.4.2. Again the proof given here relies on Itô's formula.

Proposition 10.1. Assume that $(A, \nabla)$ satisfies (LJW). Let $\Theta$ be the horizontal $\nabla^{c}$ martingale in $E$ along $X$. Then the generators of $\Theta$ and $T X^{\wedge p}$ coincide on the set of p-forms on $M$. In particular, if $\alpha$ is a harmonic $p$-form on $M$, i.e., $\langle\alpha, \Theta\rangle$ is a local martingale, then $\left\langle\alpha, T X^{\wedge p}\right\rangle$ is a local martingale. 
Proof. We only need to prove the first assertion. Since $X$ is a martingale and $(A, \nabla)$ satisfies (LJW), by Proposition 7.6, $T X^{\wedge p}$ is a $\nabla^{c}$-martingale solution to

$$
\begin{aligned}
d^{\nabla^{c}} T X^{\wedge p}= & A^{h}\left(T X^{\wedge p}\right) d B \\
& +v\left(\sum_{k=1}^{p} T X_{1} \wedge \ldots \wedge \hat{\nabla}_{T X_{k}} A(X) d B \wedge \ldots \wedge T X_{p}\right) .
\end{aligned}
$$

On the other hand, $\Theta$ satisfies

$$
d^{\nabla^{c}} \Theta=A^{h}(\Theta) d B
$$

Let $L^{\nabla}$ be the generator of $\Theta$, and let $\alpha$ be a $p$-form on $M$. The Itô equation for $\langle\alpha, \Theta\rangle$ writes

$$
d\langle\alpha, \Theta\rangle=\left\langle d \alpha, d^{\nabla^{c}} \Theta\right\rangle+\frac{1}{2} \nabla^{c} d \alpha\left(A^{h}(\Theta) d B \otimes A^{h}(\Theta) d B\right)
$$

where $d \alpha$ is the differential of $\alpha$ considered as a function on $\wedge^{p} T M$. Since $\Theta$ is a $\nabla^{c}$-martingale, the drift term in Eq. (10.3) equals $\frac{1}{2} \nabla^{c} d \alpha\left(A^{h}(\Theta) d B \otimes A^{h}(\Theta) d B\right)$. But by definition of the generator, the drift term is also equal to $L^{\nabla} \alpha(\Theta) d t$. As a consequence, we have for any $\theta \in \wedge^{p} T M$

$$
L^{\nabla} \alpha(\theta)=\frac{1}{2} \operatorname{tr} \nabla^{c} d \alpha\left(A^{h}(\theta)(\cdot) \otimes A^{h}(\theta)(\cdot)\right) .
$$

On the other hand, by means of Eq. (10.1), since $T X^{\wedge p}$ is a $\nabla^{c}$-martingale, we get the following consequence of the Itô equation for $\left\langle\alpha, T X^{\wedge p}\right\rangle$ :

$$
\begin{aligned}
& d\left\langle\alpha, T X^{\wedge p}\right\rangle \stackrel{\mathrm{m}}{=} \\
& \frac{1}{2} \nabla^{c} d \alpha\left(d T X^{\wedge p} \otimes d T X^{\wedge p}\right) \\
&=\frac{1}{2} \nabla^{c} d \alpha\left(A^{h}\left(T X^{\wedge p}\right) d B \otimes A^{h}\left(T X^{\wedge p}\right) d B\right) \\
& \quad+\nabla^{c} d \alpha\left(A^{h}\left(T X^{\wedge p}\right) d B \otimes v\left(\sum_{k=1}^{p} T X_{1} \wedge \ldots \wedge \hat{\nabla}_{T X_{k}} A(X) d B \wedge \ldots \wedge T X_{p}\right)\right) \\
& \quad+\frac{1}{2} \nabla^{c} d \alpha\left(\left(d^{\nabla^{c}} T X^{\wedge p}\right)^{\text {vert }} \otimes\left(d^{\nabla^{c}} T X^{\wedge p}\right)^{\text {vert }}\right) .
\end{aligned}
$$

Now the first line of the r.h.s. is equal to $L^{\nabla} \alpha\left(T X^{\wedge p}\right) d t$. The third line vanishes since $\alpha$ is linear on the fibers, and finally the second line vanishes since by condition (LJW), if $e \in\left(\operatorname{ker} A^{h}(\cdot)\right)^{\perp}$ then $\hat{\nabla} . A(\cdot) e=0$. We get

$$
d\left\langle\alpha, T X^{\wedge p}\right\rangle \stackrel{\mathrm{m}}{=} L^{\nabla} \alpha\left(T X^{\wedge p}\right) d t
$$

and this says that $L^{\nabla}$ coincides with the generator of $T X^{p}$ on the set of $p$-forms.

Note that Proposition 10.1 generalizes Theorem 5.2 in Malliavin [14] from 1-forms to $p$-forms. 


\section{REFERENCES}

[1] M. Arnaudon, Xue-Mei Li, and A. Thalmaier, Manifold-valued martingales, changes of probabilities, and smoothness of finely harmonic maps, Ann. Inst. H. Poincaré Probab. Statist. 35 (1999), no. 6, 765-791.

[2] M. Arnaudon and A. Thalmaier, Complete lifts of connections and stochastic Jacobi fields, J. Math. Pures Appl. (9) 77 (1998), no. 3, 283-315.

[3] __ Stability of stochastic differential equations in manifolds, Séminaire de Probabilités, XXXII, Springer, Berlin, 1998, pp. 188-214.

[4] R. Azencott, Une approche probabiliste du théorème de l'indice (Atiyah-Singer) (d'après J.-M. Bismut), Astérisque (1986), no. 133-134, 7-18, Seminar Bourbaki, Vol. 1984/85.

[5] R. O. Bauer, Characterizing Yang-Mills fields by stochastic parallel transport, J. Funct. Anal. 155 (1998), no. $2,536-549$.

[6] Yang-Mills fields and stochastic parallel transport in small geodesic balls, Stochastic Process. Appl. 89 (2000), no. 2, 213-226.

[7] N. Berline, E. Getzler, and M. Vergne, Heat kernels and Dirac operators, Springer-Verlag, Berlin, 1992.

[8] M. Campanino, Stochastic parallel displacement of tensors, Probabilistic methods in mathematical physics (Siena, 1991), World Sci. Publishing, River Edge, NJ, 1992, pp. 127-139.

[9] B. K. Driver and A. Thalmaier, Heat equation derivative formulas for vector bundles, J. Funct. Anal., to appear.

[10] K. D. Elworthy, Y. Le Jan, and Xue-Mei Li, On the geometry of diffusion operators and stochastic flows, Springer-Verlag, Berlin, 1999.

[11] M. Emery, En marge de l'exposé de Meyer "Géométrie différentielle stochastique", Séminaire de Probabilités, XVI, Supplément, Springer, Berlin, 1982, pp. 208-216.

[12] _ Stochastic calculus in manifolds, Springer-Verlag, Berlin, 1989. With an appendix by P.-A. Meyer.

[13] H. B. Lawson, Jr. and M.-L. Michelsohn, Spin geometry, Princeton University Press, Princeton, NJ, 1989.

[14] P. Malliavin, Stochastic Jacobi fields, Partial differential equations and geometry (Proc. Conf., Park City, Utah, 1977), Dekker, New York, 1979, pp. 203-235.

[15] _ Stochastic analysis, Springer-Verlag, Berlin, 1997.

[16] P.-A. Meyer, Géometrie différentielle stochastique (bis), Séminaire de Probabilités, XVI, Supplément, Springer, Berlin, 1982, pp. 165-207.

[17] J. Roe, Elliptic operators, topology and asymptotic methods, second ed., Longman, Harlow, 1998.

[18] S. Stafford, A stochastic criterion for Yang-Mills connections, Diffusion processes and related problems in analysis, Vol. I (Evanston, IL, 1989), Birkhäuser Boston, Boston, MA, 1990, pp. 313-322.

[19] K. Yano and S. Ishihara, Tangent and cotangent bundles: differential geometry, Marcel Dekker Inc., New York, 1973, Pure and Applied Mathematics, No. 16.

DÉPARTEMENT DE MATHÉmATiQUeS

Université DE Poitiers, TÉLÉPort 2 - BP 30179

F-86962 Futuroscope Chasseneuil Cedex, France

E-mail address: arnaudon@mathlabo.univ-poitiers.fr

InSTITUt FÜr ANGEWANDTE Mathematik

Universität Bonn, Wegelerstr. 6

D-53115 Bonn, GERMANY

E-mail address: anton@wiener.iam.uni-bonn.de 